\title{
Wavenumber-domain separation of rail contribution to pass-by noise
}

Article in Journal of Sound and Vibration · July 2017

DOI: 10.1016/j.jsv.2017.07.040

\section{CITATIONS}

0

6 authors, including:

Elías Zea

KTH Royal Institute of Technology

12 PUBLICATIONS 3 CITATIONS

SEE PROFILE

Some of the authors of this publication are also working on these related projects: 


\title{
Wavenumber-domain separation of rail contribution to pass-by noise
}

\author{
Elias Zea ${ }^{\mathrm{a}, *}$, Luca Manzari ${ }^{\mathrm{a}}$, Giacomo Squicciarini ${ }^{\mathrm{b}}$, Leping Feng ${ }^{\mathrm{a}}$, David Thompson ${ }^{\mathrm{b}}$, Ines Lopez \\ Arteaga ${ }^{a, c}$ \\ ${ }^{a}$ KTH Royal Institute of Technology, The Marcus Wallenberg Laboratory for Sound and Vibration Research, Teknikringen 8, \\ SE-100 44 Stockholm, Sweden \\ ${ }^{b}$ University of Southampton, Institute of Sound and Vibration Research, SO17 1BJ Southampton, United Kingdom \\ ${ }^{c}$ Eindhoven University of Technology, Department of Mechanical Engineering, 5600 MB Eindhoven, The Netherlands
}

\begin{abstract}
In order to counteract the problem of railway noise and its environmental impact, passing trains in Europe must be tested in accordance to a noise legislation that demands the quantification of the noise generated by the vehicle alone. However, for frequencies between about $500 \mathrm{~Hz}$ and $1600 \mathrm{~Hz}$, it has been found that a significant part of the measured noise is generated by the rail, which behaves like a distributed source and radiates plane waves as a result of the contact with the train's wheels. Thus the need arises for separating the rail contribution to the pass-by noise in that particular frequency range. To this end, the present paper introduces a wavenumber-domain filtering technique, referred to as wave signature extraction, which requires a line microphone array parallel to the rail, and two accelerometers on the rail in the vertical and lateral direction. The novel contributions of this research are: (i) the introduction and application of wavenumber (or plane-wave) filters to pass-by data measured with a microphone array located in the near-field of the rail, and (ii) the design of such filters without prior information of the structural properties of the rail. The latter is achieved by recording the array pressure, as well as the rail vibrations with the accelerometers, before and after the train pass-by. The performance of the proposed method is investigated with a set of pass-by measurements performed in Germany. The results seem to be promising when compared to reference data from TWINS, and the largest discrepancies occur above $1600 \mathrm{~Hz}$ and are attributed to plane waves radiated by the rail that so far have not been accounted for in the design of the filters.
\end{abstract}

Keywords: railway, noise separation, microphone array, wavenumber-domain

\section{Introduction}

The noise generated by a passing train comprises a number of sources: rolling noise, aerodynamic noise, engine noise, fan noise, among others. For trains travelling at speeds up to $300 \mathrm{~km} / \mathrm{h}$ [1] the dominant source is the rolling noise, which originates from the contact between the wheels and the rail. With the aim of legal homologation, the technical specification for interoperability (TSI) NOISE regulates the sound levels of European rail-bound vehicles to specific limit values [2]. It has however been found that a significant part of the pass-by noise comes from the track at frequencies below $2 \mathrm{kHz}$, and for this reason the compliance of a vehicle with the TSI legislation is nowadays largely dependent on the track on which such a vehicle runs. In order to facilitate the homologation process, the need thus arises for separating the track contribution to the measured pass-by noise.

The interest in the problem of pass-by noise analysis (and synthesis) is evident with the number of methods that have been developed to predict and estimate the different noise contributions. Among the

\footnotetext{
*Corresponding author. Tel. +4687907903.

Email address: zea@kth.se (Elias Zea)

$U R L:$ https://www.researchgate.net/profile/Elias_Zea (Elias Zea)
} 
synthesis methods is TWINS (Track-Wheel Interaction Noise Software) 3, 4, one of the standard prediction methods in the literature, which estimates the contributions from the wheel, rail and sleeper. TWINS takes as input roughness measurements of the wheel and rail and track decay rate measurements [5]. Another synthesis method is VAMPPASS (Vehicle Acoustic Modelling for Pass-by Prediction and Audio Simulation Software) 6, which predicts the pass-by levels by means of an equivalent source model [7, and the radiation is described by directivity patterns per equivalent source.

As regards separation (or analysis) techniques, the MISO (Multiple Input Single Output) method [8] determines an estimate of the rail contribution by means of the measured rail vibration spectra and a transfer-matrix, and the wheel contribution is inferred as the level difference between the total pass-by and the rail contribution. Another separation technique is the VTN (Vibro-acoustic Track Noise) method 9], which estimates the sound levels using source models from TWINS [10 for measured vertical and lateral rail vibrations, as well as measured sleeper vibrations. One more approach is the PBA (Pass-by Analysis) technique [11, which is a transfer-matrix method that can separate the contributions down to roughness levels, by means of knowledge of rail vibrations and sound pressure at one microphone located as defined in ISO3095 [12].

Other separation techniques include the use of an array of microphones, such as one-dimensional [13, 14 and two-dimensional 15 beamforming arrays. However, a common drawback of beamforming is that it underestimates the rail contribution by at least $10 \mathrm{~dB}$. The cause of this underestimation is that beamforming arrays are most suitable for uncorrelated sources, which is not the case for a spatially extended source such as the rail. The most recent alternative to beamforming is the so-called SWEAM (Structural Wavenumbers Estimation with an Array of Microphones) method [16, which calculates an inverse estimation of the structural wavenumbers and decay rates of the waves in the rail.

To the authors' knowledge there is no application of wavenumber filtering in the context of pass-by noise separation with microphone arrays. However, the concept of wavenumber filtering is fundamental in, for example, Fourier-based near-field acoustic holography [17, 18, in which the wavenumber spectrum of microphone array measurements is filtered in order to estimate the pressure or velocity distribution at a given distance from the array. On the other hand, wavenumber filtering is also commonly used for the identification and visualization of damage in structures, see e.g. [19, 20] and references therein, by means of measuring the wave-field with ultrasonic piezoelectric transducer arrays or with a scanning laser Doppler vibrometer.

This paper presents the wave signature extraction (WSE) method, whose purpose is to separate the rail contribution to pass-by noise by means of wavenumber-domain filters. Although the implementation presented here comprises a uniform line microphone array, the WSE method is also applicable to twodimensional microphone arrays. The main contribution of this work is the introduction and application of the rail wavenumber filters to pass-by data, without prior knowledge of the structural properties of the rail. WSE also aims at separating the total rail noise into vertical and lateral contributions, and offers the possibility of indirect estimation of the wheel contribution.

\section{Nomenclature}

$\begin{array}{ll}\kappa & \text { Acoustic wavenumber } \\ \mathbf{\Omega} & \text { Frequency vector } \\ \mathbf{\Upsilon} & \text { Vector with sub-frequencies inside a specified } 1 / 3 \text { octave band } \\ \mathbf{G}_{o}\left(\mathbf{k}, f_{c}\right) & \text { Diagonal matrix with optimal rail wavenumber filter at frequency } f_{c} \\ \mathbf{k} & \text { Wavenumber vector } \\ \mathbf{K}^{(i i)} & \text { Auto-spectrum of the } i \text {-th sensor } \\ \mathbf{K}^{(i j)} & \text { Cross-spectrum of } i \text {-th and } j \text {-th sensors } \\ \mathbf{P}(\mathbf{k}, \mathbf{\Upsilon}) & \text { Matrix with frequency-wavenumber pressure spectra } \\ \mathbf{p}\left(x_{m}, \boldsymbol{\Omega}\right) & \text { Column vector with pressure frequency spectrum of the } m \text {-th microphone } \\ \mathbf{p}_{\text {ext }}\left(\mathbf{x}, \Upsilon_{b}\right) & \text { Row vector with extrapolated pressure at } b \text {-th sub-frequency in the band } \\ \mathbf{p}_{\text {filt }}\left(\mathbf{x}, \Upsilon_{b}\right) & \text { Column vector with array pressure output of rail filter at } b \text {-th sub-frequency in the band } \\ \mathbf{s}_{i}(\mathbf{t}) & \text { Column vector with raw time-series of the } i \text {-th sensor }\end{array}$


$\mathbf{y}_{i}(\mathbf{t}) \quad$ Column vector with $1 / 3$ octave band filtered time-series of the $i$-th sensor

$\mathrm{p}_{v}^{2}, \mathrm{p}_{l}^{2} \quad$ Mean-square pressure due to vertical and to lateral rail contribution

$\mathrm{p}_{\text {filt }, v}, \mathrm{p}_{\text {filt }, l} \quad$ Root-mean square pressure output of vertical and lateral rail filter

$\phi \quad$ Plane-wave radiation angle

$\Upsilon_{b} \quad b$-th sub-frequency in the specified $1 / 3$ octave band

$B \quad$ Number of frequencies inside a specified $1 / 3$ octave band

$c \quad$ Speed of sound in air

E Number of samples of the extrapolated pressure

$f \quad$ Frequency

$f_{c} \quad$ Center frequency of a specified $1 / 3$ octave band

$k_{c}^{*} \quad$ Optimal center wavenumber at a specified $1 / 3$ octave band

$M \quad$ Number of microphones in the array

$N \quad$ Number of frequencies of the temporal Fourier transform

$w_{v}, w_{l} \quad$ Weighting factors for the vertical and lateral rail contributions

\section{Theory}

\subsection{Rail sound radiation}

A railway track consists of the rails, pads, sleepers, and ballast. The sound radiation of the sleepers dominates up to $500 \mathrm{~Hz}$, specially for a track with stiff pads [21]. Below this frequency, the rail often behaves like a compact source, and most of the sound radiation is concentrated in a small region close to the contact point with the wheel. As the frequency increases, the structural waves begin to propagate freely in the rail, and the latter behaves like a distributed source. Then the rail sound radiation becomes dominant up to about $1600 \mathrm{~Hz}$. Above this frequency it is frequently the case that the rail noise is surpassed by the wheel noise.

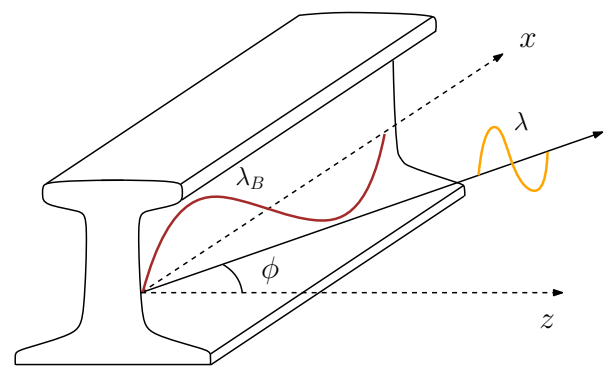

Figure 1: Sound radiation directivity of the rail. Plane waves are radiated at an angle $\phi$ in the $x-z$ plane, and $\lambda$ and $\lambda_{B}$ are the wavelengths of the acoustic and structural waves.

In the frequency range in which the rail behaves like an extended source, the sound field consists of plane waves propagating at an angle to the normal of the rail longitudinal axis. This radiation angle, lying in the $x-z$ plane, is characterized by the ratio of the acoustic wavelength and the wavelength of the bending wave that is excited in the rail. Fig. 1 shows a schematic of this situation, in which the angle follows $\phi=\arcsin \left(\lambda / \lambda_{B}\right)=\arcsin \left(k_{B} / \kappa\right)$, where $k_{B}$ and $\kappa$ are the bending and acoustic wavenumbers respectively.

During a train pass-by, the rail radiation has contributions from vertical and lateral bending waves. In general, lateral waves cut-on earlier in frequency than vertical waves because of a difference in stiffness, and there can be a total of at least four wave families up to $5 \mathrm{kHz}[22$. In order to distinguish between plane-wave families it is convenient to represent the fields in the wavenumber domain.

\subsection{Rail dispersion signature}

A complete spatio-temporal description of how plane waves propagate in a certain medium is contained in the dispersion curve. This is a plot of the wave amplitude versus wavenumber $\kappa$ and frequency $f$. For 
example, in air, the acoustic waves propagate following a linear dispersion relation $\kappa=2 \pi f / c$, where $c$ is the sound speed in air. For bending waves in a railway track, the phase velocity is frequency dependent and the dispersion curve is more sophisticated. Fig. 2 illustrates the dispersion plot obtained from a vertically excited Timoshenko beam model, and the calculated dispersion from two bogies passing in front of a microphone array. The wave in Fig. 2(a) seems to appear in Fig. 2(b), plus other waves that possibly correspond to noise radiated by the wheels and the sleepers.
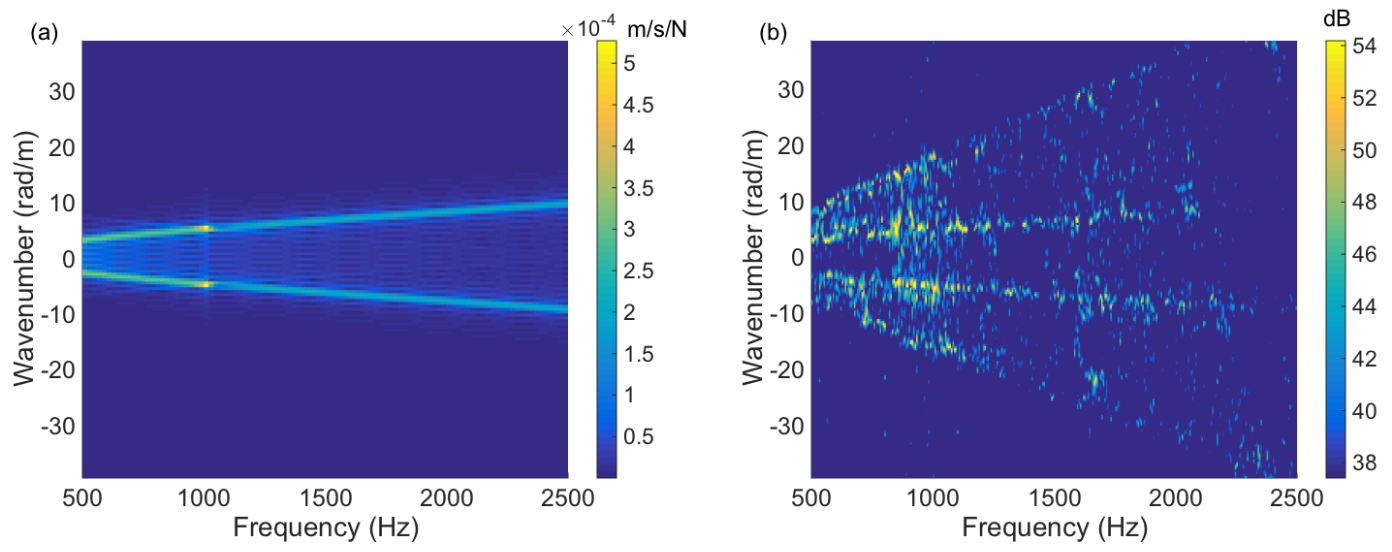

Figure 2: Dispersion plot signatures obtained from (a) vertical mobility of a periodically supported Timoshenko beam model, and from (b) array pressure measurements for a train pass-by section corresponding to two bogies running at $160 \mathrm{~km} / \mathrm{h}$.

In practice, as in Fig. 2(b), the calculation of the dispersion curve is done by means of a $2 \mathrm{D}$ discrete Fourier transform, applied to measurements taken in the space-time domain. More specifically, if the input data is a matrix with $S$ rows (spatial samples) and $T$ columns (time samples), then the 2D discrete Fourier transform operates along the rows to obtain frequency spectra, and along the columns to obtain wavenumber spectra.

It is worth mentioning that the rail dispersion on an empty track calculated from acceleration and pressure data has been previously compared in [21, for both vertical and lateral impact hammer excitations. It is shown in that study that a microphone array placed close to the track captures the acoustic plane waves radiated by the rail and the corresponding dispersion plot shows the same rail signature as the dispersion plot obtained from acceleration signals.

\section{Wave signature extraction}

The main principle of the wave signature extraction (WSE) method is to separate the rail noise by means of filtering the wavenumber spectra obtained from microphone array measurements of the train pass-by. The key point is the design and application of the wavenumber filters according to the rail signature measured with the microphone array. As mentioned above, the rail signature consists of plane waves that typically can be represented as narrow-band signals in the wavenumber domain, hence justifying the application of band-pass wavenumber filters that only accept the narrow-band content and reject the rest of the spectrum.

In general the rail signature can be estimated given the knowledge of the structural properties of the track, which would usually require a static test besides the actual train pass-by measurement. In this work, however, the rail signature is estimated from pressure and acceleration recordings taken a few seconds before and after the pass-by event, provided the microphone array is located in the near-field of the rail and two accelerometers are located on the rail: one in the vertical and one in the lateral direction. As explained in Section 3.5.1 below, this procedure to estimate the rail signature allows for the design of the band-pass wavenumber filters without the need of the additional static measurement. The application of the filters to the array pressure measured during the train pass-by thus gives the rail contribution in $1 / 3$ octave bands, as presented in Section 3.6, as well as the individual contributions due to vertical and lateral 
rail motion. As anticipated in Section 2.1 above, the applicability of the WSE method is limited to the frequency range in which the rail behaves like a spatially extended source; otherwise the rail sound radiation is rather omnidirectional and no longer consists of plane waves.

An overview of the signal processing chain from time-domain signals to the rail sound pressure levels is shown in Fig. 3, and the pseudocode of the algorithm is included in Appendix A. The remainder of this section includes a detailed explanation of each of the signals and processing blocks involved in the WSE method.

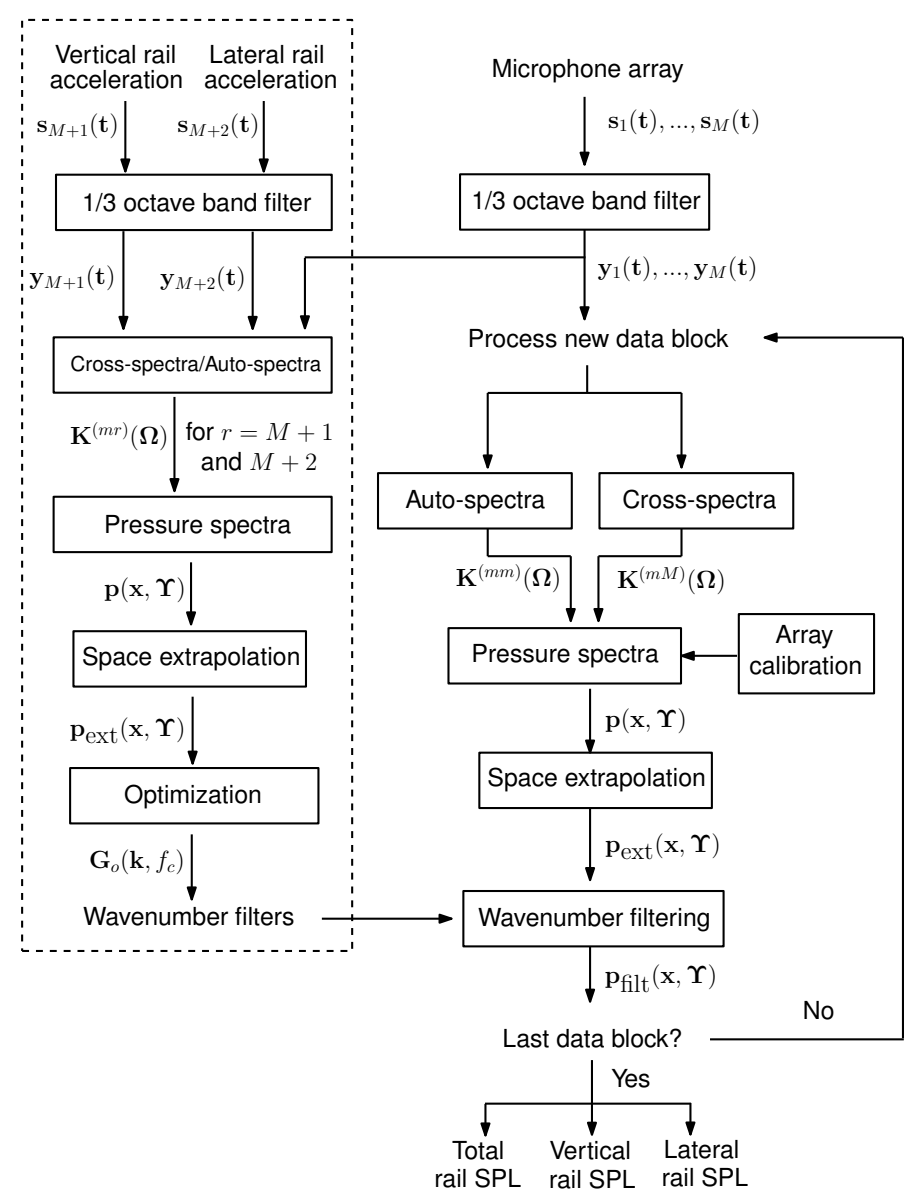

Figure 3: Block diagram of the wave signature extraction method. The dashed box comprises the estimation of the optimal filter with data before and after the train pass-by.

As depicted in Fig. 3, the inputs are time-domain signals, in units of volts, from an $M$-channel line microphone array, and vertical and lateral rail vibrations measured by two accelerometers in front of the array. Let us assume each signal is recorded during the time vector $\mathbf{t} \in \mathbb{R}^{L}$, and we group them in a matrix of raw measurements

$$
\mathbf{S}(\mathbf{t})=\left[\begin{array}{lllll}
\mathbf{s}_{1}(\mathbf{t}) & \mathbf{s}_{2}(\mathbf{t}) & \cdots & \mathbf{s}_{M+1}(\mathbf{t}) & \mathbf{s}_{M+2}(\mathbf{t})
\end{array}\right] \in \mathbb{R}^{L \mathrm{x}(M+2)} .
$$

\subsection{1/3 octave band filtering}

In the context of noise calculations, it is customary to provide results in $1 / 3$ octave bands. In order to account for this in the WSE method, the first signal processing stage entails the application of a 1/3 octave band filter to the raw data measured with all sensors. This filter outputs time-domain signals that only 
contain the frequency content of a chosen band centered at a frequency $f_{c}$. The filter is a digital Butterworth band-pass filter $\mathbf{h}(\boldsymbol{\tau})$, and it has length $D<L$. Let us define the filtered matrix as

$$
\mathbf{Y}(\mathbf{t})=\left[\begin{array}{lllll}
\mathbf{y}_{1}(\mathbf{t}) & \mathbf{y}_{2}(\mathbf{t}) & \cdots & \mathbf{y}_{M+1}(\mathbf{t}) & \mathbf{y}_{M+2}(\mathbf{t})
\end{array}\right] \in \mathbb{R}^{L \mathrm{x}(M+2)}, \quad \text { where } \quad \mathrm{y}_{i}\left(t_{l}\right)=\sum_{d=1}^{D} \mathrm{~h}\left(t_{l}-\tau_{d}\right) \mathrm{s}_{i}\left(\tau_{d}\right) .
$$

\subsection{Spectral estimation}

Since the end results of the WSE method comprise rail contributions versus frequency, it becomes natural to transform the temporal data to the frequency domain. Therefore the auto-spectrum of every microphone in the array is estimated, as well as the cross-spectrum between every microphone and a given reference signal. At this point the signals are divided into overlapping blocks of length $N$, and the rest of the processing is repeated per block unless otherwise stated. Let us define the power spectrum in units of $\mathrm{V}^{2}$, for arbitrary sensors $i$ and $j$, as the diagonal matrix $[1]$

$$
\mathbf{K}^{(i j)}(\boldsymbol{\Omega})=\frac{\varsigma}{N^{2}} \operatorname{diag}\left\{\mathbf{V} \mathbf{U y}_{i}(\mathbf{t})\right\} \operatorname{diag}\left\{\mathbf{V U} \mathbf{y}_{j}(\mathbf{t})\right\}^{H} \in \mathbb{C}^{N \times N},
$$

where $\boldsymbol{\Omega} \in \mathbb{R}^{N}$ is the frequency vector in $\mathrm{Hz}$, the operator $\mathbf{V} \in \mathbb{C}^{N \mathrm{x} N}$ is the $1 \mathrm{D}$ temporal discrete Fourier transform matrix, the diagonal matrix $\mathbf{U}$ has an $N$-length time window function on its elements, the superscript $H$ denotes Hermitian transpose, and $\varsigma$ is the windowing correction factor. It is worth mentioning that, in practice, the discrete Fourier transform is preferably performed via fast Fourier transform algorithms such as Cooley-Tukey [23] instead of via multiplication with the Fourier matrix.

Then the auto-spectrum of the $m$-th microphone equals $\mathbf{K}^{(\mathrm{mm})}(\boldsymbol{\Omega})$, whilst the cross-spectrum is computed with respect to the reference signal and it follows $\mathbf{K}^{(m r)}(\boldsymbol{\Omega})$, where, for instance, $r=M+1$ and $r=M+2$ for vertical and lateral acceleration signals respectively. For the sake of brevity we shall develop the equations for an arbitrary reference signal $r$, since it is only a matter of choosing $r$. Furthermore, the computational time for the entire WSE algorithm can be halved by means of processing one-sided spectra, that is, taking only the first $N / 2+1$ frequencies in $\boldsymbol{\Omega}$. In that case Eq. (3) must then have a multiplicative factor of 2 .

\subsection{Pass-by pressure spectra}

The following processing block is to transform the microphone array data from units of Volts to Pascals and account for the phase information between the microphones, such that a complex pressure signal is obtained in the space-frequency domain. The pressure spectrum of the $m$-th microphone, in units of $\mathrm{Pa}$, is provided by the $N$-length vector

$$
\mathbf{p}\left(x_{m}, \boldsymbol{\Omega}\right)=\chi_{m} \mathrm{e}^{-\mathrm{j} \boldsymbol{\Theta}_{m r}},
$$

where $\mathrm{j}=\sqrt{-1}, \boldsymbol{\Theta}_{m r}(\boldsymbol{\Omega})$ is the phase of $\mathbf{K}^{(m r)}$ in radians, and the vector

$$
\chi_{m}(\boldsymbol{\Omega})=\frac{1}{\zeta_{m}} \mathbf{c}_{m}\left[\mathbf{K}^{(m m)}\right]^{1 / 2}
$$

follows, where $\mathbf{c}_{m}(\boldsymbol{\Omega}) \in \mathbb{C}^{N}$ is the calibration transfer function in units of $\mathrm{Pa} / \mathrm{Pa}$ for the $m$-th microphone (see Section 4 for measurement details), and $\zeta_{m} \in \mathbb{R}^{+}$is the nominal sensitivity of the $m$-th microphone in units of $\mathrm{V} / \mathrm{Pa}$. The pressure spectra in the space-frequency domain are then grouped in matrix form as

$$
\mathbf{P}(\mathbf{x}, \boldsymbol{\Omega})=\left[\begin{array}{llll}
\mathbf{p}\left(x_{1}, \boldsymbol{\Omega}\right) & \mathbf{p}\left(x_{2}, \boldsymbol{\Omega}\right) & \cdots & \mathbf{p}\left(x_{M}, \boldsymbol{\Omega}\right)
\end{array}\right] \in \mathbb{C}^{N \times M} .
$$

\footnotetext{
${ }^{1}$ The superscript $(i j)$ does not denote an element of the matrix, but rather that the matrix contains the frequency spectra for the $i$-th and $j$-th sensors in its diagonal.
} 


\subsection{Spatial data extrapolation}

Spatial extrapolation of microphone array data is a common technique used in near-field acoustic holography (see e.g. 24), in order to extend the spatial aperture (or length) of the measurement, hence improve the resolution in the wavenumber domain when applying the spatial Fourier transform. In the context of the WSE method, the space-frequency pressure matrix in Eq. (6) is extrapolated along its rows. This extrapolation is only applied to the $B$ rows corresponding to the frequencies inside the $1 / 3$ octave band centered at $f_{c}$. Here linear predictive border padding [25] is employed, which estimates the additional spatial samples by means of fitting an auto-regressive model to the measured pressure field.

Let us define the set of frequencies inside the band centered at $f_{c}$ as $\boldsymbol{\Upsilon}=\left\{\Upsilon_{1}, \Upsilon_{2}, \ldots, \Upsilon_{B}\right\} \subset \boldsymbol{\Omega}$. Then, the padding algorithm finds the coefficients $\alpha_{m}\left(\Upsilon_{b}\right)$ required to solve the difference equation

$$
\operatorname{p}_{\text {ext }}\left(x_{\mu}, \Upsilon_{b}\right)=-\sum_{m=1}^{M} \alpha_{m}\left(\Upsilon_{b}\right) \mathrm{p}\left(x_{\mu-m}, \Upsilon_{b}\right), \quad \text { for } \quad \mu=M+1, M+2, \ldots, \frac{E+M}{2},
$$

at the $b$-th frequency in the band, where $E-M$ is even and corresponds to the amount of extrapolated sample:2 This equation is solved towards both ends of the array length, and for all frequencies in $\boldsymbol{\Upsilon}$. The resulting pressure fields are organized in a matrix

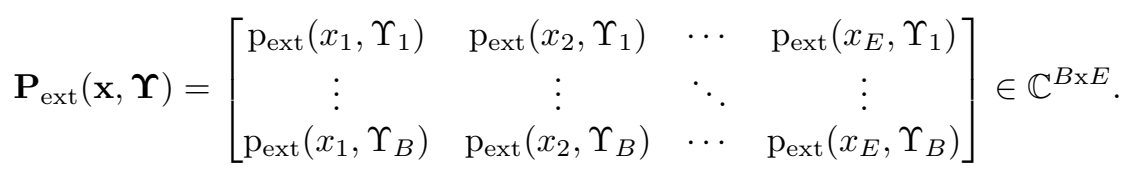

\subsection{Rail wavenumber filters}

The previous sections have presented the required signal processing blocks used to prepare the raw measurement data for filtering in the wavenumber domain. The novelty of this paper is the design and application of band-pass wavenumber filters to the array measurements in order to separate the rail contribution to the total pass-by noise. It is only this part of the method in which the data measured with the accelerometers is used, for the sake of obtaining an accurate description of the vertical and lateral rail signatures and design the filters accordingly. The following includes the filter design and digital implementation.

\subsubsection{Design basis and considerations}

The ideal grounds to design a wavenumber filter for the sound field radiated by the rail is from microphone array measurements on an empty track. The rail signature can then be found by means of performing an impact hammer test for both vertical and lateral excitations, and the filters can be tailored to only pass the wavenumbers that show up in the dispersion curve. In this way, the filters yield only the sound fields radiated by the vertical and lateral rail vibrations. Nevertheless, this impact test measurement can in principle be avoided because the speed of the bending waves in the rail is about five times higher than the speed of sound in air, thus it is expected that the rail begins to radiate sound some seconds before the train is actually in front of the array ${ }^{3}$. Similarly, once the train has passed, the rail keeps radiating sound for some seconds. The significance of this fact is that the information before and after the pass-by can be used to design the rail filters, such that these are applied to the sound fields during the pass-by. Moreover, specially for high train speeds, Doppler shift may influence the filter functions if the rail signature is obtained from static tests instead of with dynamic (pass-by) tests; thus using information before/after the pass-by might be a more desirable choice. An additional result found in [21 that further motivates this strategy is that the rail signatures of an empty track and a loaded track are nearly identical in the frequency range of interest.

\footnotetext{
${ }^{2}$ In Eq. 77), the subscript $\mu$ refers to the index of the extrapolated spatial sample, that is, sample $\mu=(E-M) / 2+1$ is equivalent to sample $m=1$ in Eq. 6). Also, the indexes $\mu=M+1$ and $M+2$ ought not to be associated with the acceleration signals here.

${ }^{3}$ The number of seconds depends on the train speed and the track decay rates.
} 
In general, empty track measurements are performed separately with vertical and lateral hammer excitations, yet the case of a train pass-by exhibits both excitations (and sometimes coupling between them). Thus, if we aim at accurately capturing, for example, the vertical rail signature, it might be reasonable to use the data corresponding to the time in which the vertical vibrations are stronger than the lateral vibrations. In other words, let us define a signal power ratio (SPR) per frequency band and per time block as

$$
\operatorname{SPR}\left(f_{c}, \mathrm{t}_{j}\right)=10 \log _{10} \frac{\operatorname{tr} \mathbf{K}^{(v v)}(\mathbf{\Upsilon})}{\operatorname{tr} \mathbf{K}^{(l l)}(\mathbf{\Upsilon})}, \quad \text { for } \mathrm{t}_{j} \text { before/after the pass-by, }
$$

where $v=M+1$ and $l=M+2$ correspond to vertical and lateral acceleration signals. Then, the input data that can be used for the design of the vertical filter corresponds to the time blocks $t_{j}$ for which SPR $>0$ $\mathrm{dB}$. Likewise, for the lateral filter, the time blocks for which SPR $<0 \mathrm{~dB}$ can be taken. If the SPR is zero for a given block, the data can be disregarded and the next block is evaluated.

\subsubsection{Filter design optimization}

The separation accuracy of the WSE method depends on the filter parameters: center wavenumber $\mathrm{k}_{c}$ and pass-band $\mathrm{k}_{\mathrm{pb}}$; hence it is imperative to have a means for choosing these values appropriately. To preserve conciseness only the process for the vertical filter is described. Also, the blocks of time-data are restricted to the aforementioned conditions on the SPR. Let us begin with the wavenumber-frequency domain representation of Eq. (8):

$$
\mathbf{P}(\mathbf{k}, \mathbf{\Upsilon})=\left[\begin{array}{lll}
\mathbf{p}\left(\mathbf{k}, \Upsilon_{1}\right) & \cdots & \mathbf{p}\left(\mathbf{k}, \Upsilon_{B}\right)
\end{array}\right] \in \mathbb{C}^{\operatorname{Ex} B},
$$

where $\mathbf{p}\left(\mathbf{k}, \Upsilon_{b}\right)=\mathbf{Q} \mathbf{W} \mathbf{p}_{\text {ext }}^{T}\left(\mathbf{x}, \Upsilon_{b}\right) / E$, with superscript $T$ denoting vector transpose, $\mathbf{W} \in \mathbb{R}^{E x E}$ a diagonal matrix containing a spatial Tukey window, and $\mathbf{Q} \in \mathbb{C}^{E x E}$ the $1 \mathrm{D}$ spatial discrete Fourier transform matrix. This representation is equivalent to the pressure dispersion curve within the frequency band $\boldsymbol{\Upsilon}$. Here, the $e$-th element of the wavenumber vector $\mathbf{k}$ is defined as

$$
\mathrm{k}_{e}=[2(e-1)-E] \frac{\Delta k}{2} \text { for } \quad e=1, \ldots, E,
$$

where $\Delta k=2 \pi /(E \Delta x)$ is the wavenumber resolution and $\Delta x$ is the microphone spacing, and the highest alias-free wavenumber is $\pi / \Delta x$.

With the purpose of promoting computational efficiency, the wavenumber domain can be restricted according to two facts: (i) the waves before the pass-by have a negative (positive) wavenumber if the $+x$ axis is defined against (towards) the train direction, and (ii) the waves we are after are not evanescent. Therefore, provided for instance that the $+x$ axis is defined against the train direction and data before the pass-by is used, the wavenumber domain can be restricted to the triangular region $-2 \pi \mathbf{\Upsilon} / c \prec \mathbf{k} \prec 0$. At a given frequency $\Upsilon_{b}$ in the band, this region can be understood as all propagating waves, $\mathrm{k}_{z}=\sqrt{\kappa^{2}-\mathrm{k}_{e}^{2}} \in \mathbb{R}$, with $\kappa=2 \pi \Upsilon_{b} / c$ and $e \in[1, E / 2]$.

The proposal is then to estimate the center wavenumber of the filter with an optimization algorithm, grounded on the assumption that before/after the pass-by only the rail is radiating noise, thus the situation is closest to that of an empty track. If this assumption is valid, then the optimal filter should have the pass-band centered at the maximum of the pressure wavenumber spectrum. It is also assumed that there is at most one prominent bending wave for vertical and for lateral rail vibration. (The case of more than one wave per direction introduces a layer of complexity that is out of the scope of this paper.) Then, at a given frequency $\Upsilon_{b}$, the optimization algorithm can be formulated in the wavenumber domain as

$$
\mathrm{k}_{c}\left(\Upsilon_{b}\right)=\underset{\mathbf{k}}{\arg \max }\left|\mathbf{H}_{\text {pre }}\left(\mathbf{k}, f_{c}\right) \mathbf{p}\left(\mathbf{k}, \Upsilon_{b}\right)\right| \quad \text { subject to } \quad \begin{cases}-2 \pi f_{c} / c \prec \mathbf{k} \prec 0 & \text { before pass-by or } \\ 0 \prec \mathbf{k} \prec 2 \pi f_{c} / c & \text { after pass-by, }\end{cases}
$$

where the diagonal matrix $\mathbf{H}_{\text {pre }}\left(\mathbf{k}, f_{c}\right)$ is a pre-conditioning band-pass Butterworth filter. The pass-band of this conditioning filter corresponds to $\mathbf{k} \in\left[1, \beta \pi f_{c} / c\right]$, in which the cut-on wavenumber discards plane 
waves propagating with normal incidence, that is $\mathrm{k}_{e} \rightarrow 0$, and the cut-off discards monopole- and dipole-like sources that manifest as plane waves propagating at grazing incidence [26], that is $\left|\mathrm{k}_{e}\right| \rightarrow \kappa$. Here the value of $\beta$ has been found empirically, and equals 0.75 and 1.5 for vertical and lateral filters.

The functional in Eq. 12 is not necessarily convex, since at some frequencies there might be contributions from both vertical and lateral rail vibrations to the measured sound fields. Therefore an elementby-element approach ought to be adopted to find the maximum. This is solved for the $B$ frequencies in the band $f_{c}$, and the optimal center wavenumber $\mathrm{k}_{c}^{*}\left(f_{c}\right)$ is found from the median of the set $\mathbf{k}_{c}(\mathbf{\Upsilon})$. The preference of the median instead of mean operator here is that it guarantees that the center wavenumber is exactly one of the wavenumbers of the set $\mathbf{k}_{c}(\mathbf{\Upsilon})$. Appendix B includes the pseudocode of this algorithm.

On the other hand, the pass-band of the filter $\mathrm{k}_{\mathrm{pb}}$ is left as a tuning parameter. It is worth considering that the spatial decay rate of the track has a frequency dependent behavior for vertical and lateral rail vibrations, in which oftentimes the vertical decay is higher than the lateral decay for frequencies below 1 $\mathrm{kHz}$, and the opposite can happen above $2 \mathrm{kHz}[22$. Due to the duality property of the Fourier transform, a higher decay (or a smaller bandwidth) in the spatial domain is equivalent to a larger bandwidth in the wavenumber domain. We propose then to take this information into account at the moment of choosing the pass-bands of the vertical and lateral filters.

It is important to stress that the optimal center wavenumber is not necessarily equal to the actual rail bending wavenumber. However the center wavenumber accounts for any effects associated to wavenumber sampling rate (or array length), thus it can be expected that the more microphones are used, the closer the optimal center wavenumber will be to the bending wavenumber.

\subsubsection{Digital implementation}

The choice of IIR Butterworth filter families is adopted in this paper. Let us denote the optimal filter at the frequency band $f_{c}$, for instance for the vertical rail contribution, as the diagonal matrix $\mathbf{G}_{o}\left(\mathbf{k}, f_{c}\right) \in$ $\mathbb{C}^{E \mathrm{x} E}$. Its magnitude response has a pass-band $\mathrm{k}_{\mathrm{pb}}$, and the latter is centered at $\mathrm{k}_{c}^{*}$. The pass-band choice rule is a larger bandwidth for the vertical filter than for the lateral filter. The phase response must be zero-phase such that the spatial phase of the input sound field is preserved. In order to account for possible wavenumber shifts, the filters are constructed by means of setting: (i) the filter response obtained with data before the pass-by in the negative wavenumber axis, and (ii) the filter response obtained with data after the pass-by in the positive wavenumber axis.

The output of the vertical filter within the frequency band $f_{c}$ can then be grouped in a matrix

$$
\mathbf{P}_{\text {filt }}(\mathbf{x}, \mathbf{\Upsilon})=\left[\begin{array}{lll}
\mathbf{p}_{\text {filt }}\left(\mathbf{x}, \Upsilon_{1}\right) & \cdots & \mathbf{p}_{\text {filt }}\left(\mathbf{x}, \Upsilon_{B}\right)
\end{array}\right] \in \mathbb{C}^{E \mathrm{x} B}
$$

where the $b$-th column of the matrix follows from zero-phase filtering the extrapolated sound field:

$$
\mathbf{p}_{\text {filt }}\left(\mathbf{x}, \Upsilon_{b}\right)=\mathbf{Q}^{H} \mathbf{G}_{o}^{H}\left(\mathbf{k}, f_{c}\right) \mathbf{G}_{o}\left(\mathbf{k}, f_{c}\right) \mathbf{Q W} \mathbf{p}_{\mathrm{ext}}^{T}\left(\mathbf{x}, \Upsilon_{b}\right)
$$

Here the input sound field is obtained via Eqs. (4)-(7) with $r=M$. This procedure is analogously performed with the lateral filter. It is worth stressing that because the filters have zero-phase response their outputs have the same spatial phase as the input (extrapolated pressure field).

\subsection{Rail contributions to the sound pressure level}

Once the filters have been applied to the microphone array pressure during the pass-by, the next and final step is to calculate the total rail, vertical rail and lateral rail contributions to the sound pressure level at a given microphone position. Firstly, the total rail contribution is calculated from the outputs of the vertical and lateral filters. At a given position in the array $x_{\mu}$ and within the $1 / 3$ octave band $f_{c}$, the root-mean-square (RMS) pressure obtained from the output of the vertical filter is defined as

$$
\mathrm{p}_{\text {filt }, v}\left(x_{\mu}, f_{c}\right)=\sqrt{\delta_{f} \frac{1}{\pi B} \sum_{b=1}^{B}\left|\mathrm{p}_{\mathrm{filt}}\left(x_{\mu}, \Upsilon_{b}\right)\right|^{2}}
$$


where the dimensionless variable $\delta_{f}$ is the octave bandwidth per unit $\mathrm{Hz}$, and one-sided spectra is used. A similar calculation is done for the RMS output of the lateral filter: $\mathrm{p}_{\mathrm{filt}, l}\left(x_{\mu}, f_{c}\right)$. Then the total rail contribution to the sound pressure level (SPL) in dBA follows

$$
\operatorname{SPL}_{\text {rail }}\left(x_{\mu}, f_{c}\right)=20 \log _{10}\left[\frac{\mathrm{p}_{\text {filt }, v}\left(x_{\mu}, f_{c}\right)+\mathrm{p}_{\text {filt }, l}\left(x_{\mu}, f_{c}\right)}{\mathrm{p}_{\text {ref }}}\right]+A\left(f_{c}\right),
$$

where $\mathrm{p}_{\text {ref }}=20 \mu \mathrm{Pa}$ and $A\left(f_{c}\right)$ is the A-weighting filter coefficient at $f_{c}$.

This way of calculating the total rail contribution must be equivalent to the energetic sum of the vertical and lateral rail contributions. In mathematical terms, let us define the mean-square pressure of these contributions as

$$
\begin{aligned}
\mathrm{p}_{v}^{2} & =\mathrm{p}_{\text {filt }, v}^{2}+w_{v} \mathrm{p}_{\text {filt }, v} \mathrm{p}_{\text {filt }, l}, \\
\mathrm{p}_{l}^{2} & =\mathrm{p}_{\text {filt }, l}^{2}+w_{l} \mathrm{p}_{\text {filt }, l} \mathrm{p}_{\text {filt }, v},
\end{aligned}
$$

where $w_{v}$ and $w_{l}$ are weighting factors that follow $w_{v}+w_{l}=2$. The latter condition establishes the equivalence between Eq. (16) and the energetic sum of the individual rail contributions. The choice here is to set $w_{v}=w_{l}=1$, which means that both contributions are equally important to the total rail contribution. Then, the vertical (and similarly lateral) rail contribution to the SPL in dBA follows:

$$
\mathrm{SPL}_{\mathrm{vert}}\left(x_{\mu}, f_{c}\right)=10 \log _{10}\left[\frac{\mathrm{p}_{\text {filt }, v}^{2}+\mathrm{p}_{\text {filt }, v} \mathrm{p}_{\text {filt }, l}}{\mathrm{p}_{\text {ref }}^{2}}\right]+A\left(f_{c}\right) .
$$

At this point the algorithm checks whether the last data block has been reached (see Fig. 3). If it is the last block, the algorithm stops and calculates the rail contributions. Otherwise the previous stages are repeated with the new block of data. The calculation of the rail contributions once all blocks have been processed comprises averaging the SPL values per block obtained during the iterative process.

\section{Experimental setup}

The measurement campaign was carried out in a high-speed track with standard ballast, near Munich, Germany, in June 2016. The experimental setup is shown in Figs. 4 and 5. It consists of a 42-channel line microphone array, two accelerometers and a pass-by microphone located $7.5 \mathrm{~m}$ away from the track center as defined in ISO3095 12. The array consists of 6 GRAS 40BD and 36 GRAS 40PH microphones. The acceleration sensors are ICP accelerometers B\&K 4398, and the pass-by microphone is a BSWA Tech MA201. All sensors are connected to a VXI multi-channel acquisition system, and the time signals are simultaneously sampled at $6.4 \mathrm{kHz}$.

The microphone array is positioned $1.2 \mathrm{~m}$ away from the nearest rail, and at half the rail web in height. The array is held on an aluminium frame, which rests on three supports fixed with ballast. The microphone spacing is $8 \mathrm{~cm}$, which gives an array length of $3.36 \mathrm{~m}$. Windshields are used in order to minimize the influence of flow in the measurements. The accelerometers are mounted with magnets on the rail, and located in front of the left-most microphone of the array. One accelerometer is placed in the vertical direction underneath the rail, and the other is placed in the lateral direction at half the rail web. Two train passages are considered: one at $80 \mathrm{~km} / \mathrm{h}$ and one at $160 \mathrm{~km} / \mathrm{h}$.

In order to fully account for the scattering in the measurements due to the microphone array, the latter is calibrated in free-field in an anechoic chamber at the Marcus Wallenberg Laboratory for Sound and Vibration Research in KTH. A monopole sound source is used to produce an omnidirectional field, and the distances from the microphones to the source are measured. Then the calibration transfer functions $\left\{\mathbf{c}_{1}(\boldsymbol{\Omega}), \ldots, \mathbf{c}_{M}(\boldsymbol{\Omega})\right\}$ used in Eq. (5) (see Section 3.3) are the result of dividing the analytical monopole sound field at the microphone positions by the pressure measured at these positions. 


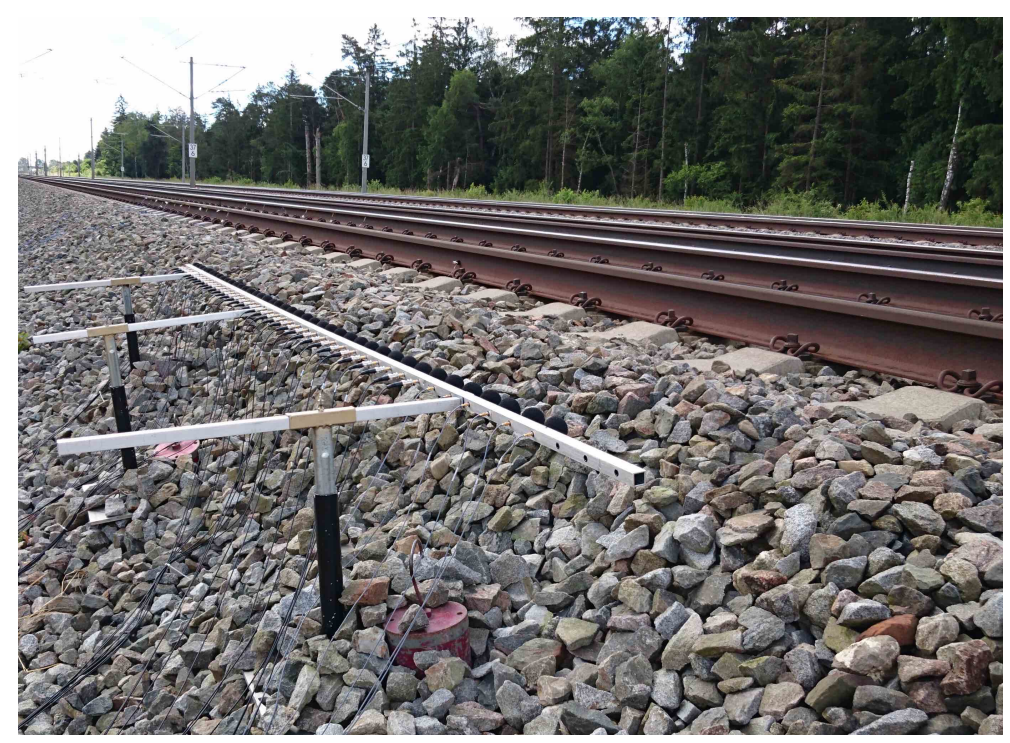

Figure 4: Photograph of the experimental setup at the track section in Germany.

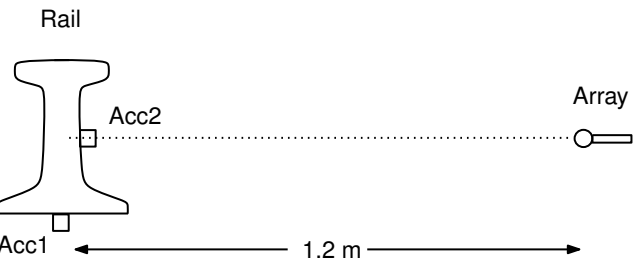

Figure 5: Schematic (side-view) of the experimental setup in Germany.

\section{Results}

\subsection{Dispersion signatures before and after the pass-by}

This section includes dispersion signatures obtained with recordings of about 30 seconds before and after the pass-by. The dispersion is calculated with respect to the vertical and lateral acceleration signals. The temporal block size is $N=4096$ samples, which gives a frequency resolution of $\Delta f \approx 1.56 \mathrm{~Hz}$, and $95 \%$ overlap is used between blocks. The extrapolated array space consists of $E=210$ samples, which gives a wavenumber resolution of $\Delta k=0.374 \mathrm{rad} / \mathrm{m}$.

Fig. 6 shows the pressure dispersion signatures before the pass-by of the train running at $160 \mathrm{~km} / \mathrm{h}$. It can be seen that the plane waves propagate with a negative wavenumber, and this is physically meaningful since the $+x$ axis is defined against the train direction. It can also be observed that, as expected, the vertical wave appears to have a smaller wavenumber than the lateral wave, due to the different stiffness of the rail in these directions 22. In Fig. 6(a), however, it appears as if the lateral wave is being captured by the array even when referencing the spectra with respect to vertical acceleration, and this indicates that there might be a coupling of the lateral wave with the vertical rail vibrations. It is also noticeable the presence of other dispersive waves which look like replicas of the strongest wave, and are separated by approximately $10.5 \mathrm{rad} / \mathrm{m}$. These replicas are due to periodicity effects of the sleeper spacing, and the fact that there are not as many replicas in Fig. 6(b) is an indicator of the difference in bending stiffness [22]. Moreover, there is another wave with negative wavenumber and higher slope than the vertical and lateral waves in Fig. 6(a), and this corresponds to the air dispersion curve $\kappa=-2 \pi f / c$ possibly related to plane waves propagating at grazing incidence. It is worth stressing that, since the array is located in the near-field and it is of finite length, it is very difficult to separate sources which radiate plane waves at grazing incidence. In equivalent 

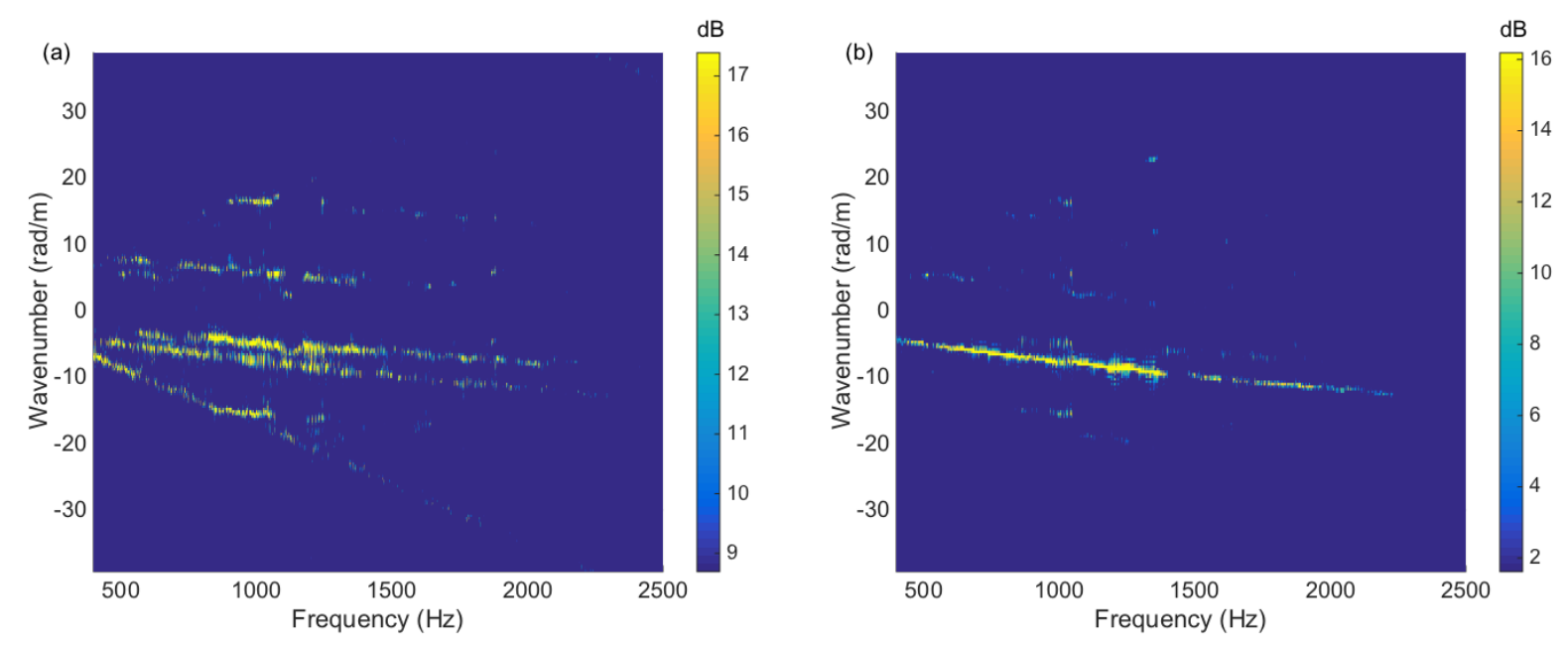

Figure 6: Dispersion plot signatures from Eq. 10 expressed in $\mathrm{dB}$ re $20 \mu \mathrm{Pa}$, and obtained with pressure spectra referenced to (a) vertical and (b) lateral acceleration signals, using data before the pass-by.

words, the array becomes "blind" to such sources and groups them as a unique plane wave at $\kappa=-2 \pi f / c$ in the dispersion curve.

Fig. 7 shows the dispersion signatures after the pass-by. Since the train has passed, the waves now propagate with a positive wavenumber, and a clear symmetry can be seen when compared to Fig. 6 . Replicas are also visible in Fig. 7(a), as well as the air dispersion curve yet now following the relationship $\kappa=2 \pi f / c$. Overall these results indicate that the assumption of the rail radiating noise before and after the pass-by holds, and that the filters can be designed with this data.
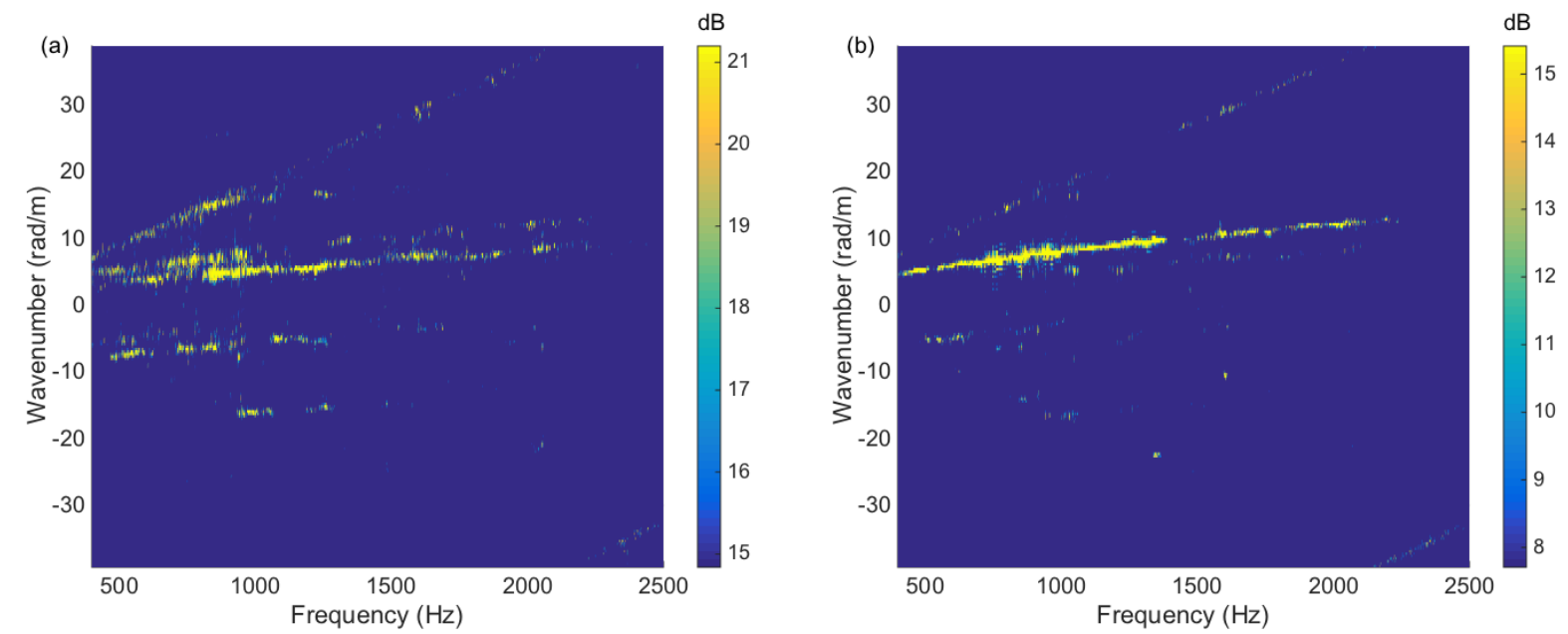

Figure 7: Dispersion plot signatures from Eq. 10 expressed in $\mathrm{dB}$ re $20 \mu \mathrm{Pa}$, and obtained with pressure spectra referenced to (a) vertical and (b) lateral acceleration signals, using data after the pass-by.

For the sake of illustration, Fig. 8 shows the SPR versus measurement time at $1000 \mathrm{~Hz}$, obtained with data before and after the pass-by, and for both train speeds. In Fig. 8 (a), corresponding to $80 \mathrm{~km} / \mathrm{h}$ train speed, the vertical vibrations dominate for about 3 to 4 seconds before and after the pass-by, and the lateral vibrations dominate during the remaining time blocks. On the other hand, the time intervals during which the vertical vibrations dominate in Fig. 8(b) are slightly smaller than those in Fig. 8(a), which reveals a dependence of the SPR with the train speed as anticipated in Section 3.5.1 
(a)

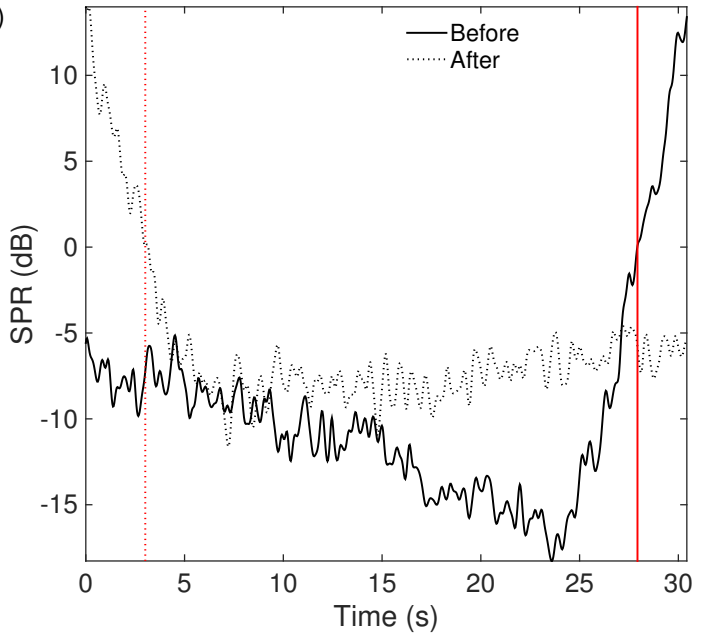

(b)

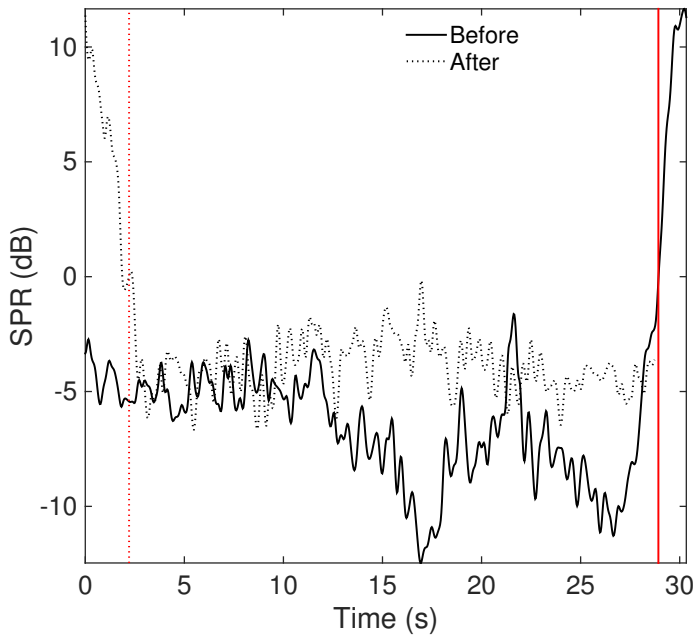

Figure 8: Signal power ratio from Eq. 9 at $1000 \mathrm{~Hz}$, using 30 seconds of data before and after the pass-by. The train speed is (a) $80 \mathrm{~km} / \mathrm{h}$ and (b) $160 \mathrm{~km} / \mathrm{h}$. The vertical solid (dashed) lines indicate the time at which the SPR becomes negative when using data before (after) the pass-by.

\subsection{Filter optimization}

Results of the optimization algorithm are shown in this section. Table 1 includes the optimal center wavenumbers obtained with data before and after the pass-by. The train speed is $160 \mathrm{~km} / \mathrm{h}$, yet for 80

Table 1: Optimal vertical and lateral center wavenumbers obtained with data before and after the pass-by, and analytical bending wavenumbers of a free Timoshenko beam obtained with measurements used for reference data.

\begin{tabular}{|c|c|c|c|c|c|c|}
\hline \multirow{2}{*}{$\begin{array}{l}\text { Frequency } \\
(\mathrm{Hz})\end{array}$} & \multicolumn{3}{|l|}{ Vertical } & \multicolumn{3}{|l|}{ Lateral } \\
\hline & $\begin{array}{l}\mathrm{k}_{c}^{*} \text { before } \\
(\mathrm{rad} / \mathrm{m})\end{array}$ & $\begin{array}{l}\mathrm{k}_{c}^{*} \text { after } \\
(\mathrm{rad} / \mathrm{m})\end{array}$ & $\begin{array}{l}\text { Timoshenko } \\
(\mathrm{rad} / \mathrm{m})\end{array}$ & $\begin{array}{l}\mathrm{k}_{c}^{*} \text { before } \\
(\mathrm{rad} / \mathrm{m})\end{array}$ & $\begin{array}{l}\mathrm{k}_{c}^{*} \text { after } \\
(\mathrm{rad} / \mathrm{m})\end{array}$ & $\begin{array}{l}\text { Timoshenko } \\
(\mathrm{rad} / \mathrm{m})\end{array}$ \\
\hline 500 & -2.99 & 2.99 & 3.33 & -5.05 & 5.24 & 5.00 \\
\hline 630 & -3.74 & 3.74 & 3.80 & -5.98 & 6.17 & 5.65 \\
\hline 800 & -4.30 & 4.49 & 4.36 & -6.73 & 6.55 & 6.40 \\
\hline 1000 & -5.05 & 5.05 & 5.02 & -7.85 & 7.67 & 7.26 \\
\hline 1250 & -5.98 & 5.42 & 5.82 & -9.16 & 9.16 & 8.27 \\
\hline 1600 & -7.11 & 7.11 & 6.79 & -10.29 & 10.66 & 9.44 \\
\hline 2000 & -7.67 & 8.23 & 7.98 & -11.59 & 11.97 & 10.83 \\
\hline
\end{tabular}

$\mathrm{km} / \mathrm{h}$ the results are nearly identical. The center wavenumbers agree well with the fact that the vertical stiffness is greater than the lateral stiffness, thus vertical wavenumbers are smaller than lateral wavenumbers. The comparison of the wavenumbers before and after the pass-by indicates a small shift at some frequencies, and this is attributed to inexactitudes of the optimization algorithm. For the sake of comparison, the wavenumbers of a free Timoshenko beam representing the rail are also reported in the table. To obtain these, the vertical and lateral bending stiffnesses are set to $6.4 \mathrm{MN} \cdot \mathrm{m}^{2}$ and $1.07 \mathrm{MN} \cdot \mathrm{m}^{2}$ and the shear coefficient is set to 0.4 [27]. The effect of the pad-sleeper-ballast support is not included for simplicity, but it is assumed to be small in this frequency range due to the low stiffness of the rail pads fitted in this track (this was estimated to be at around $100 \mathrm{MN} / \mathrm{m}$, as it will be shown below). These analytically calculated wavenumbers are in good agreement with those estimated with the measurements, and this comparison confirms that the optimization procedure can extract the correct wavenumbers for the filters without knowledge of the structural properties of the rail. 
Fig. 9 shows the center wavenumbers of Eq. (12) (per band sub-frequency) that are used to determine the optimal center wavenumber of the vertical filter. Data after the pass-by is used in Fig. 9(a), and data before the pass-by is used in Fig. 9(b). In Fig. 9(a) the vertical center wavenumbers inside the frequency
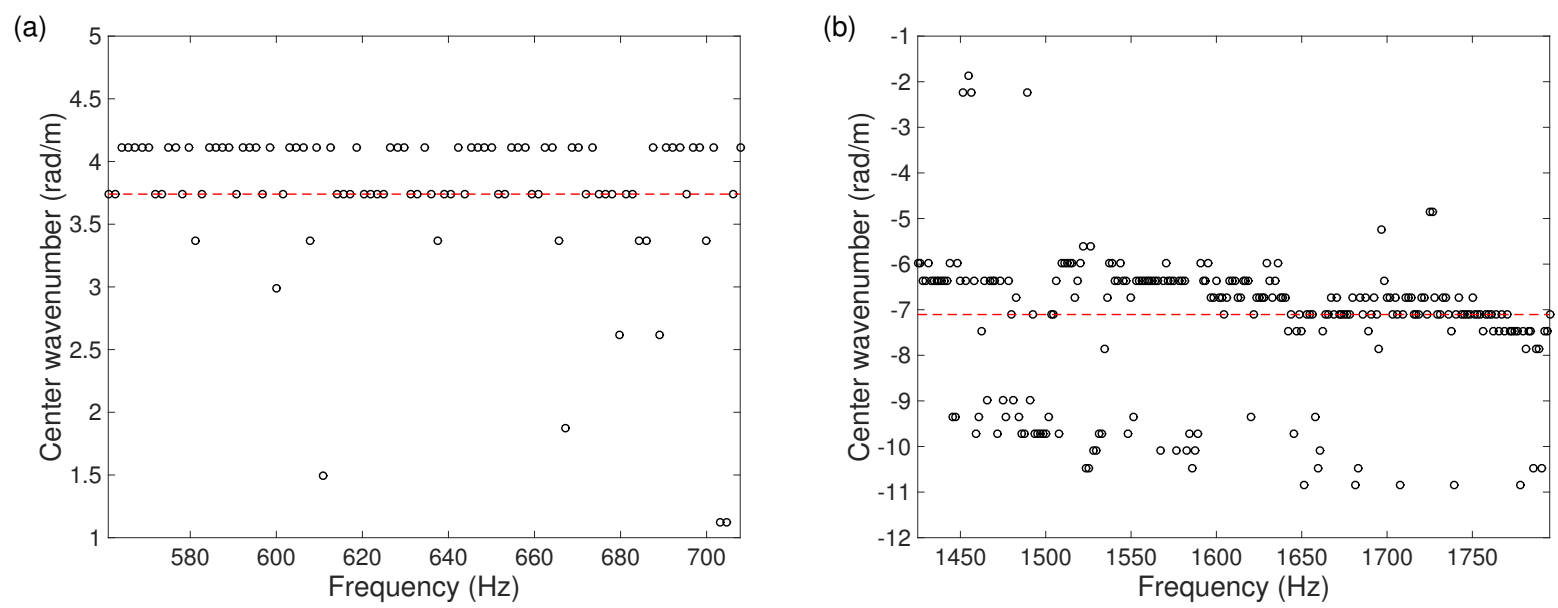

Figure 9: (a) Circles: set of vertical center wavenumbers obtained from Eq. 12 in the frequency band centered at $630 \mathrm{~Hz}$, using data after the pass-by. Dashed-line: optimal center wavenumber $\mathrm{k}_{c}^{*}$. (b) Same as (a) but at $1600 \mathrm{~Hz}$ and using data before the pass-by.

band mostly oscillate between $3.7 \mathrm{rad} / \mathrm{m}$ and $4.1 \mathrm{rad} / \mathrm{m}$, yet a few times are smaller than these values. The use of the median eliminates the influence of these outliers and yields the value of $3.74 \mathrm{rad} / \mathrm{m}$. Similarly in Fig. 9(b), the optimal value is around $-7.1 \mathrm{rad} / \mathrm{m}$, however the curve exhibits a negative slope versus frequency. This slope can also be seen in Fig. 6(a), and is attributed to the fact that the $+x$ axis is defined against the train direction hence the plane waves travel with negative wavenumber before the pass-by. It can also be noticed in Fig. 9(b) that, at some frequencies, it seems as if there is another wave present with a more negative wavenumber, which likely corresponds to the lateral wave (see Fig. 6(a)). In addition, the reason for the higher density of circles in Fig. 9(b) is that the frequency band centered at $1600 \mathrm{~Hz}$ has a larger amount of sub-frequencies than the band centered at $630 \mathrm{~Hz}$.

The resulting filters are illustrated in Fig. 10 at $800 \mathrm{~Hz}$ and $2000 \mathrm{~Hz}$, using the center wavenumbers from Table 1. The pass-band is $2.5 \mathrm{rad} / \mathrm{m}$ for the vertical filter and $1.5 \mathrm{rad} / \mathrm{m}$ for the lateral filter. The order
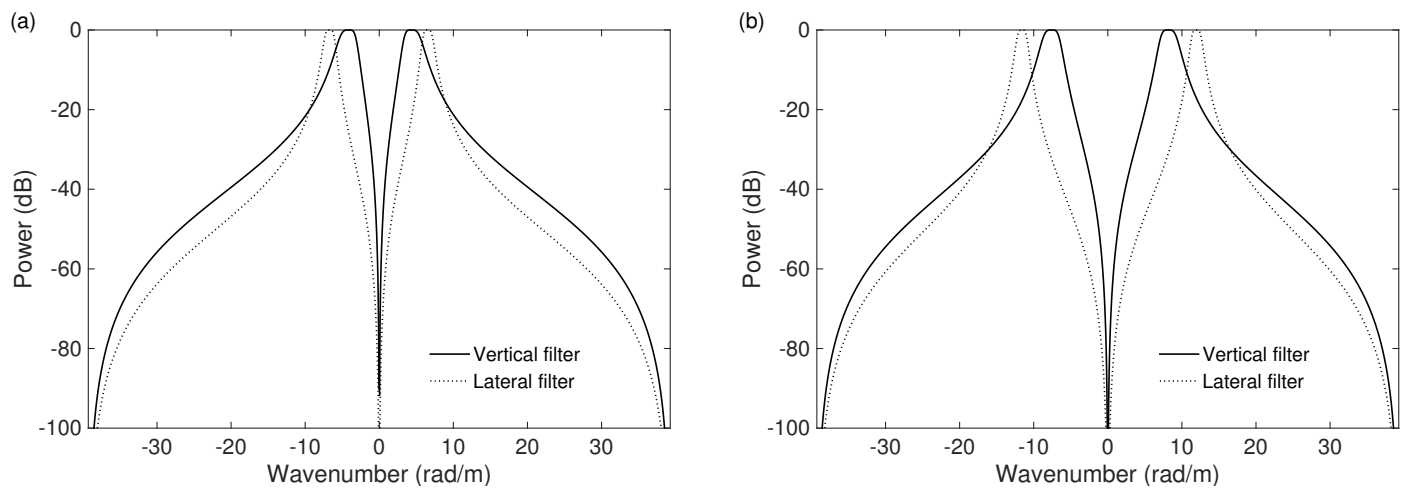

Figure 10: Power of the optimal vertical and lateral filters at (a) $800 \mathrm{~Hz}$ and (b) $2000 \mathrm{~Hz}$ in the wavenumber domain.

of the filters is 4 , and these have a power of $-3 \mathrm{~dB}$ at the cut-on and cut-off wavenumbers. Nonetheless it is worth pointing out that the application of zero-phase filtering via Eq. (14) duplicates the power of the filters shown in Fig. 10 . 


\subsection{Application of wavenumber filters}

The result of applying the rail filters to pass-by data in the wavenumber domain is shown in this section. The block size is $N=1024$, with $95 \%$ overlap, and the extrapolated space consists again of $E=210$ samples. The reference sensor chosen here is the left-most microphone in the array, that is, $r=M$ in Eq. (4). The time window of analysis during the pass-by is shown in Fig. 11, and it corresponds to the passage of two bogies and four wheel sets: approximately $26 \mathrm{~m}$ long.

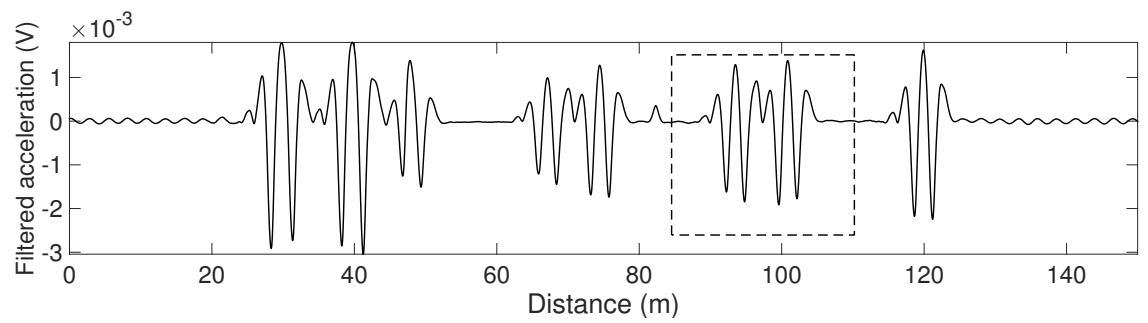

Figure 11: Solid curve: band-pass filtered vertical acceleration, in which the minima correspond to the wheels passing above the accelerometer. Dashed box: analysis window of length of one vehicle, corresponding to two bogies and four wheel sets. The distance axis is the measurement time axis multiplied with the train speed.

SPL snapshots for both train speeds at three time blocks are shown in Figs. 12 and 13 , illustrating the filtering procedure at 1000 and $1600 \mathrm{~Hz}$ respectively. The wavenumber axis is zoomed into the propagating wave region, i.e. $|\mathbf{k}|<\kappa$, in order to have a clearer view of the filtering process. The time instance during the analysis window is also shown on each snapshot. Making an overall comparison of the two train

(a)

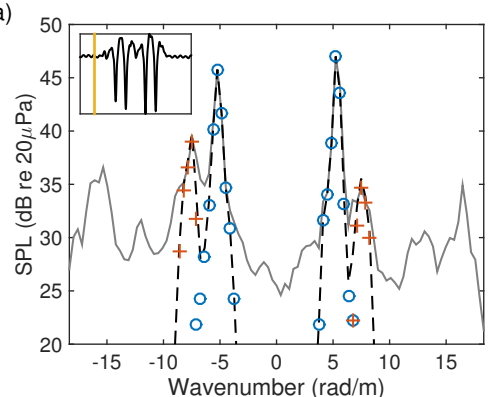

(d)

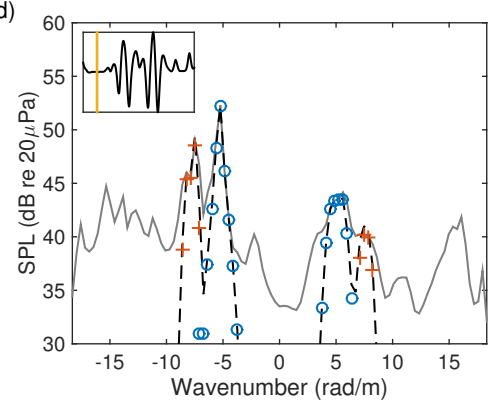

(b)

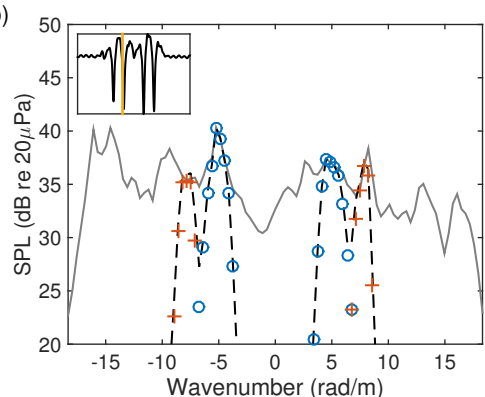

(e)

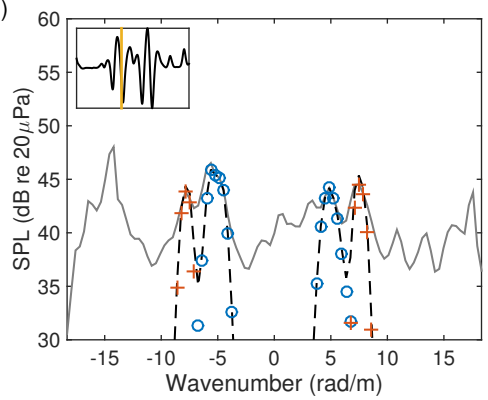

(c)

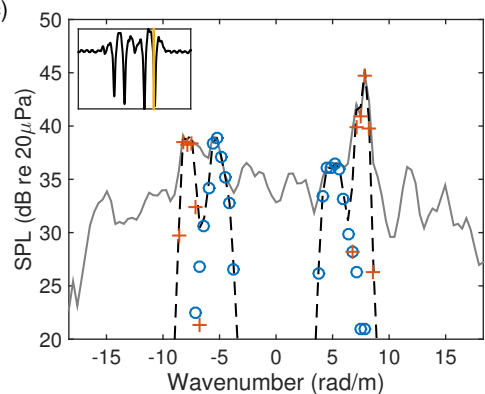

(f)

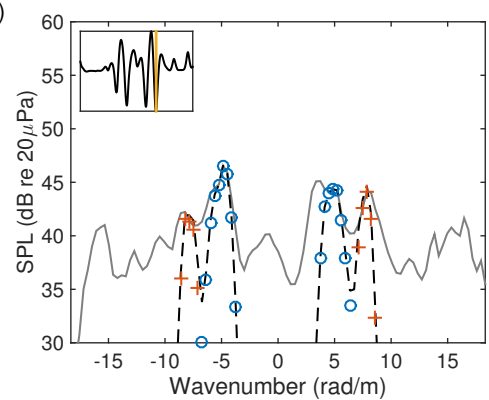

Figure 12: Top row: wavenumber-domain sound pressure levels of the total pass-by noise (-), total rail noise (- -), vertical rail noise $(\mathrm{o})$ and lateral rail noise $(+)$, for the train running at $80 \mathrm{~km} / \mathrm{h}$, and the frequency band centered at $1000 \mathrm{~Hz}$. Bottom row: same as top row but with the train running at $160 \mathrm{~km} / \mathrm{h}$. The box in the north-west region of each subfigure contains the analysis window, and its corresponding time instance depicted with a vertical yellow line. The minima of the curve in this box correspond with the passage of the wheels above the accelerometer positioned in the vertical direction. 
speeds, it can be noticed that the SPL of the faster train is higher than that of the slower train. Besides the plane waves radiated by the rail there is a broadband wavenumber content, which may correspond to the omnidirectional-like radiation of the wheel in the spatial domain [10. More into detail, the sound field radiated by the vertical wave is almost always stronger than that radiated by the lateral wave, which indicates that the vertical rail contribution is likely greater than the lateral rail contribution in this frequency band. This happens for both train speeds, and it can be interpreted as that the excitation direction of the wheel/rail contact is predominantly of vertical nature. In addition, as seen in Figs. 12(a) and (d) as well as 13 (a) and (d), it is expected that the total rail contribution is greater in the time instances at which the wheels are furthest from the array.
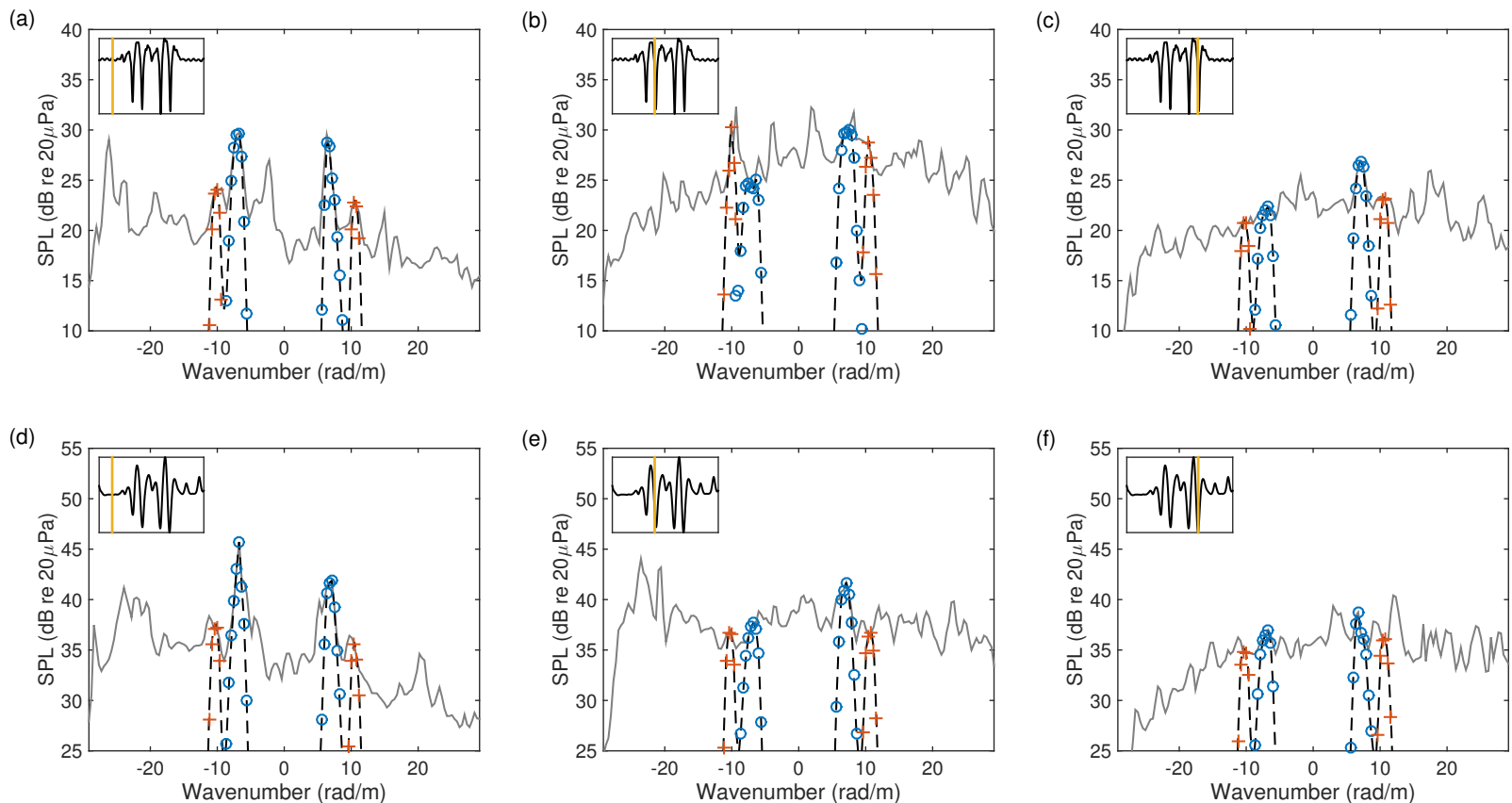

Figure 13: Top row: wavenumber-domain sound pressure levels of the total pass-by noise (-), total rail noise (- -), vertical rail noise (o) and lateral rail noise $(+)$, for the train running at $80 \mathrm{~km} / \mathrm{h}$, and the frequency band centered at $1600 \mathrm{~Hz}$. Bottom row: same as top row but with the train running at $160 \mathrm{~km} / \mathrm{h}$. The box in the north-west region of each subfigure contains the analysis window, and its corresponding time instance depicted with a vertical yellow line. The minima of the curve in this box correspond with the passage of the wheels above the accelerometer positioned in the vertical direction.

On the other hand, the wavenumber domain can also be interpreted as the domain of plane--wave incidence angles, by means of using the relation $\phi=\arcsin (\mathbf{k} / \kappa)$. Considering the $+x$ axis is defined against the train direction, then the time instances at which the wheel/rail contact is on the right-hand side of the array are expected to exhibit a stronger wavenumber content for waves propagating with negative incidence angles (or, equivalently, with negative wavenumbers $\mathrm{k}_{e}$ ). Similarly, the wavenumber content is expected to be stronger for waves propagating with positive incidence angles when the wheel/rail contact is on the left-hand side of the array. This phenomenon is most noticeable in Figs. 13(d) and (f).

In Figs. 13(a) and (c), as well as in Figs. 13(d) and (f), the broadband content appears to have opposite slopes versus wavenumber, and this is attributed to the position of the four wheels with respect to the array at the given time instances. Additionally, in Figs. 13(a) and (b) there is a substantial contribution of a plane wave centered at a little less than $5 \mathrm{rad} / \mathrm{m}$, which may correspond to the sound field radiated by an additional bending wave in the rail. 


\subsection{Description of reference data}

In order to provide verification of the WSE technique against a different approach, noise predictions are presented in this section based on the TWINS model [4. This allows the different noise components to be calculated separately; namely the radiation from the sleeper, rail vertical motion, rail lateral motion and wheel. The procedure has been validated through field experiments [4. In this case, to increase the reliability of the source separation, measured vibration levels of the rail and of the sleepers are adopted as an intermediate input in the model. In [28] it is shown that the uncertainty is significantly reduced when measured vibration is adopted. Therefore the procedure followed to predict the noise components consists of the following three steps: (1) a "standard" TWINS modelling of the measurement situation is first performed, (2) the predicted sound power levels from step 1 are then compensated using measured track vibration, and finally (3) all the components are adjusted so that the total predicted noise matches the measured noise spectrum. The results in the second step are equivalent to using the radiation models for the track directly with measured vibration spectra. No adjustment is made to the wheel component in this step. In the last step the relative contribution of each component in each frequency band is retained. Correcting the predicted spectra in step 3 is only acceptable if the difference between measured and predicted noise at step 2 is small. It is assumed in performing step 3 that the relative contributions of wheel and track are correct in each frequency band so the final adjustment is only to adjust the contributions of each band to the A-weighted noise level. This is also necessary to allow a comparison with the WSE method where the summation of the different components always corresponds exactly to measured noise spectrum.

Direct measurements of rail and wheel roughness and of decay rates are available and are shown in Fig. 14. Fig. 14(a) also includes an analytical estimate of decay rates using a model of a Timoshenko beam on a continuous two-layer support 29. This best fitting line is obtained with a pad stiffness in the vertical direction of $100 \mathrm{MN} / \mathrm{m}$. This low value of stiffness is responsible for the decay rate being low in a wide frequency range, resulting in strong noise radiation from the rail.
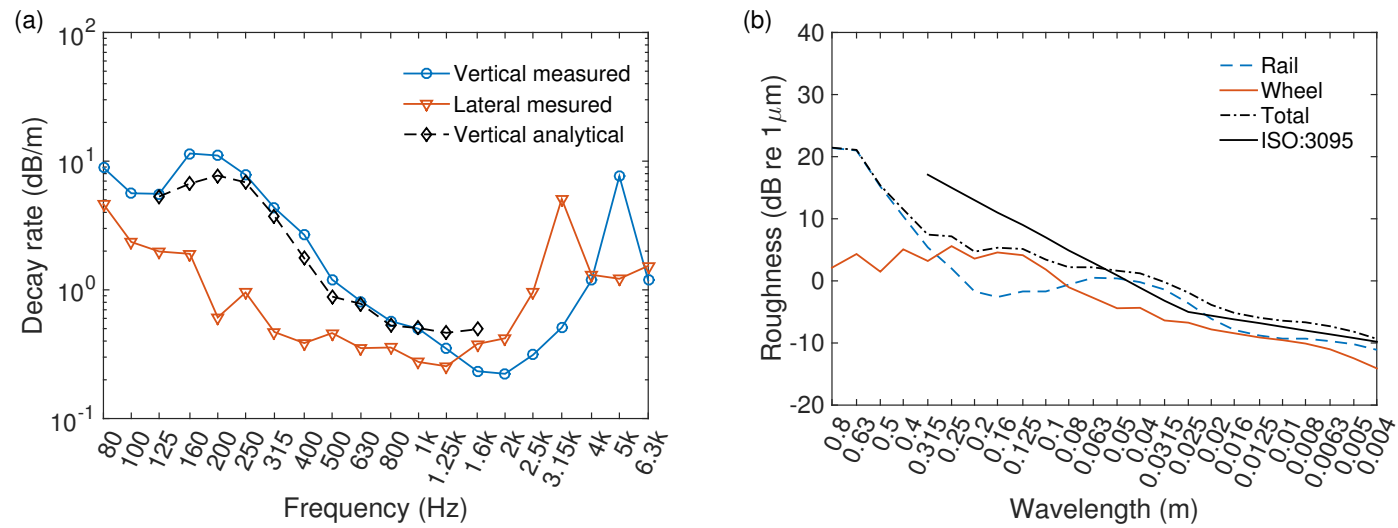

Figure 14: (a) Decay rates and (b) roughness for the test site used for TWINS predictions of the reference data.

The other important parameters required for the noise calculation are listed in Table 2, The rail crossreceptance factor [29] has been adjusted to give the best fit between the lateral rail vibration and the measured spectra. The ballast stiffnesses are default values that have not been tuned to the current case. The measured track decay rates shown in Fig. 14 are used in the predictions.

The wheel is modelled using finite elements to obtain modal parameters and then mobilities via a modal summation approach. The modal frequencies and damping ratios have been tuned to match mobilities measured with impact hammer tests.

As an example of the procedure adopted to obtain the various components, Fig. 15 shows the noise spectra as obtained in steps 2 and 3 for the train speed of $80 \mathrm{~km} / \mathrm{h}$. The results are expressed as sound pressure spectra in one-third octave bands corresponding to the $\mathrm{L}_{\mathrm{Aeq}}$ over the $26 \mathrm{~m}$ analysis window (shown 
Table 2: Track parameters used for TWINS calculations.

\begin{tabular}{llll}
\hline Track component & Parameter & Vertical & Lateral \\
\hline Rail & Bending stiffness $\left(\mathrm{MN} \cdot \mathrm{m}^{2}\right)$ & 6.42 & 1.07 \\
& Damping loss factor & 0.02 & 0.02 \\
& Mass per unit length $(\mathrm{kg} / \mathrm{m})$ & 60 & \\
Railpad & Cross-receptance factor $(\mathrm{dB})$ & -10 & 13.6 \\
& Stiffness $(\mathrm{MN} / \mathrm{m})$ & 100 & 0.2 \\
Sleeper & Damping loss factor & 0.2 & Constant mass \\
& Model & Modal & 140 \\
Ballast & Mass $(\mathrm{kg})$ & - & 0.6 \\
& Spacing $(\mathrm{m})$ & 0.6 & Constant \\
& Model & Frequency-dependent & 35 \\
& Stiffness $(\mathrm{MN} / \mathrm{m})$ & - & 2 \\
\hline
\end{tabular}

in Fig. 11 in the dashed box). Measurements and calculations are presented for a receiver set at $7.5 \mathrm{~m}$ from the nearest rail and $1.5 \mathrm{~m}$ above the top of rail. This does not correspond to one of the microphones in the WSE array but it is close to the TSI reference position at $7.5 \mathrm{~m}$ from the centre of the track. Similar results are found for different receiver positions. To allow for propagation above a reflecting ground, the Delany \& Bazley model [30] with flow resistivity of $105 \mathrm{~Pa} \cdot \mathrm{s} / \mathrm{m}^{2}$ is adopted in the calculation.

(a)

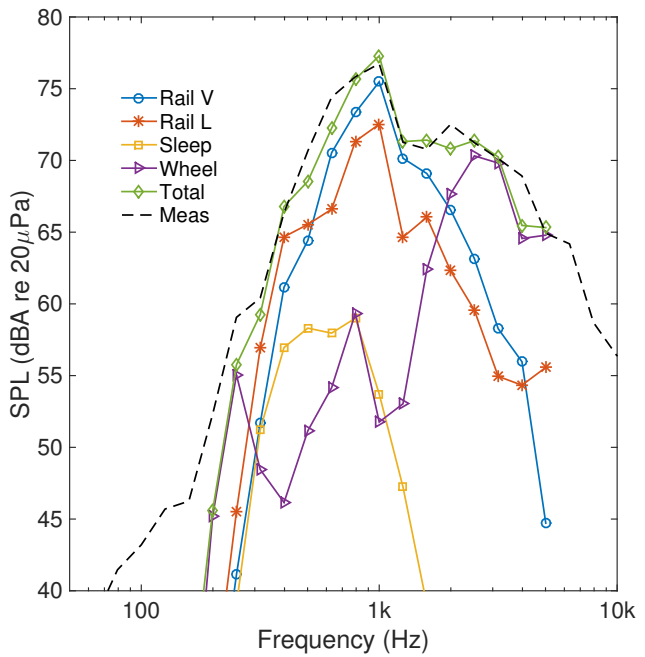

(b)

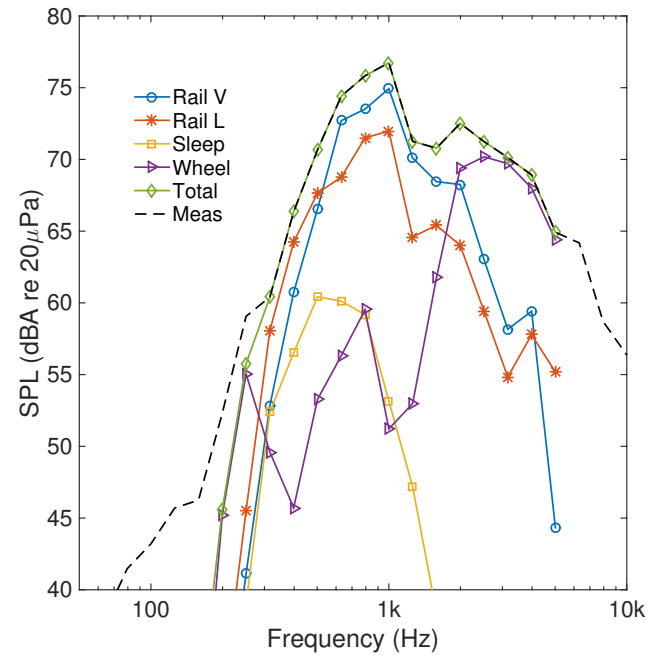

Figure 15: TWINS predictions after (a) step 2 and (b) step 3. In Step 2 sound power levels are compensated from track vibration measurements while in step 3 components are adjusted to match measured spectra. These predictions are performed in order to produce the reference data, and are independent of the measured data used in the WSE method.

The results obtained at step 2, Fig. 15(a), are already in good agreement with the measurements. Between $315 \mathrm{~Hz}$ and $5 \mathrm{kHz}$ the average difference is $1 \mathrm{~dB}$ and the maximum is $3 \mathrm{~dB}$; the final corrections of step 3 can be safely applied, see Fig. 15(b). The rail is the predominant source between $315 \mathrm{~Hz}$ and 1.2 $\mathrm{kHz}$ and also dominates the overall noise. The wheel contribution equals that of the rail at $2 \mathrm{kHz}$ and is dominant at higher frequency. The same procedure is applied to predict results at the position of the array and at $160 \mathrm{~km} / \mathrm{h}$. The rail component predictions for these cases are shown in the next section where they are compared with the WSE separation results. 


\subsection{Validation of the WSE method}

The predictions made with TWINS, described in the above section, are used as reference data. The microphone $m=21$ ( or $\mu=E / 2+1$ ) is used for the comparison.

\subsubsection{Separation of total rail contribution}

Figs. 16 and 17 show the SPL spectra of the total rail noise for train speeds of 80 and $160 \mathrm{~km} / \mathrm{h}$ respectively. Firstly it can be stated that the rail spectra is very close to the total pass-by spectra, thus
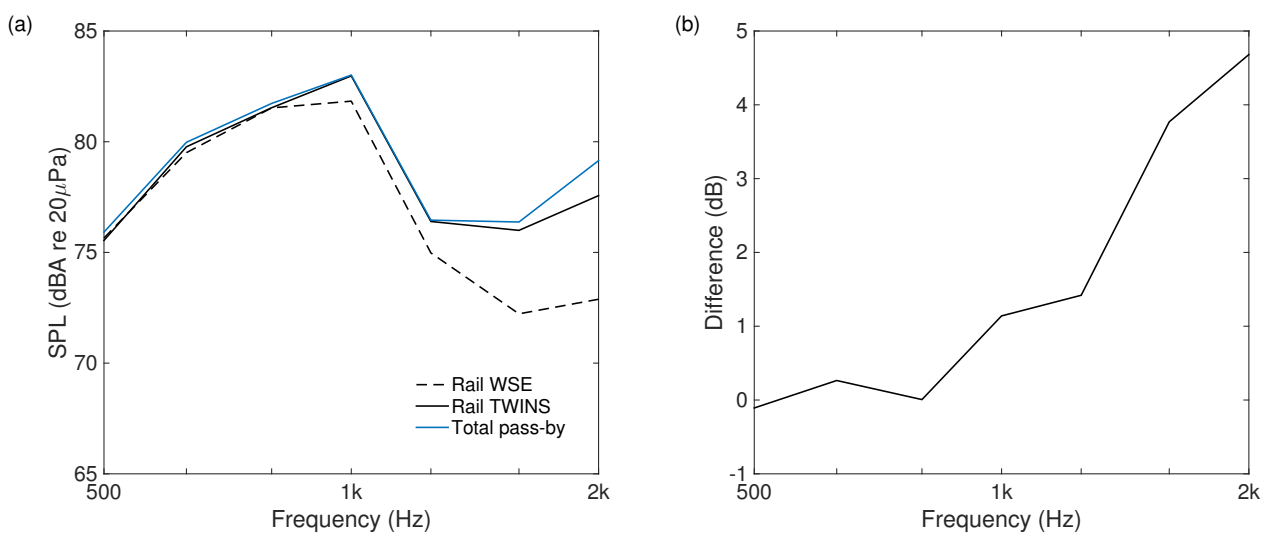

Figure 16: (a) Sound pressure level spectra of the rail contribution for the train running at $80 \mathrm{~km} / \mathrm{h}$, predicted by TWINS and the WSE method. The total pass-by is that predicted by TWINS. (b) Difference between WSE and TWINS results of rail contribution.

there is a significant contribution from the rail in the measured pressure in this frequency range. Overall the rail contribution obtained with the WSE method agrees well with the predictions from TWINS. However, an underestimation of the rail contribution by the WSE method can be seen in Fig. 16 at 1600 and 2000 $\mathrm{Hz}$, with a magnitude of 3.8 and $4.7 \mathrm{~dB}$ respectively. In Fig. 17 the underestimation is not as significant,
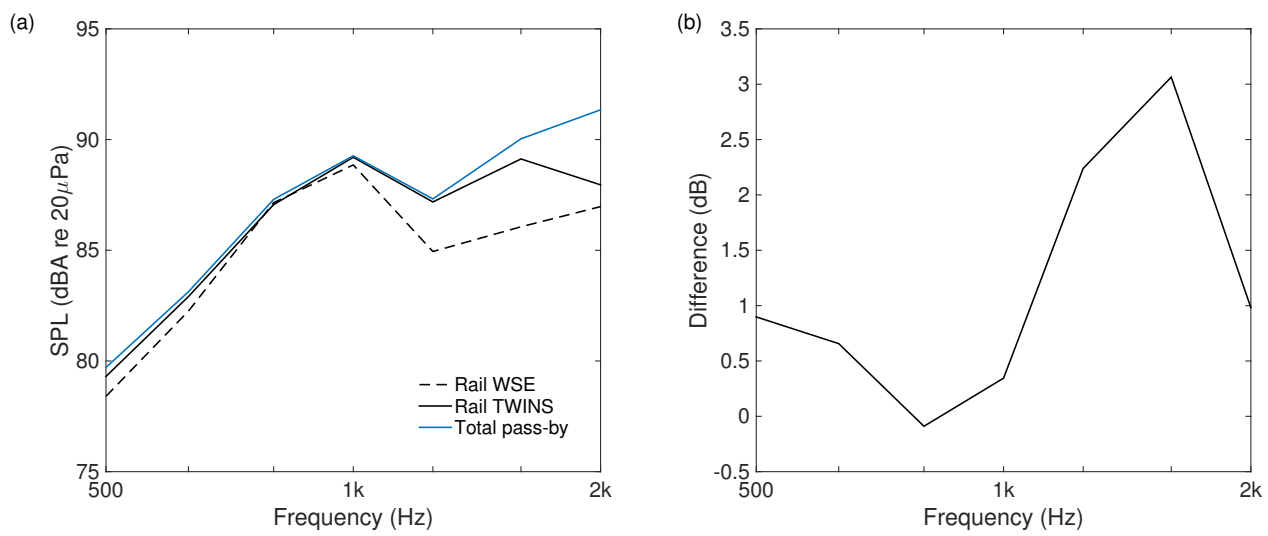

Figure 17: (a) Sound pressure level spectra of the rail contribution for the train running at $160 \mathrm{~km} / \mathrm{h}$, predicted by TWINS and the WSE method. The total pass-by is that predicted by TWINS. (b) Difference between WSE and TWINS results of rail contribution.

with a magnitude of $3 \mathrm{~dB}$, and is most noticeable at $1600 \mathrm{~Hz}$.

One of the results found in Section 5.3 is that, at $1600 \mathrm{~Hz}$, there appears to be an additional acoustic plane wave which might correspond to a secondary bending wave (see Figs. 13(a) and (b)), yet it is disregarded by the filtering process. At present it is considered that this is a web bending wave, as described in [22]. 
Provided this acoustic wave is indeed radiated by the rail, its filtering could explain the underestimation of the total rail noise. In what follows the separation of vertical and lateral rail components is presented.

\subsubsection{Separation of vertical and lateral rail contributions}

Figs. 18 and 19 illustrate the SPL spectra of the vertical and lateral rail contributions for train speeds of 80 and $160 \mathrm{~km} / \mathrm{h}$ respectively. The results obtained from the WSE method are compared with the predictions obtained from TWINS. The difference in dB between the two methods are also shown for each of the rail components.
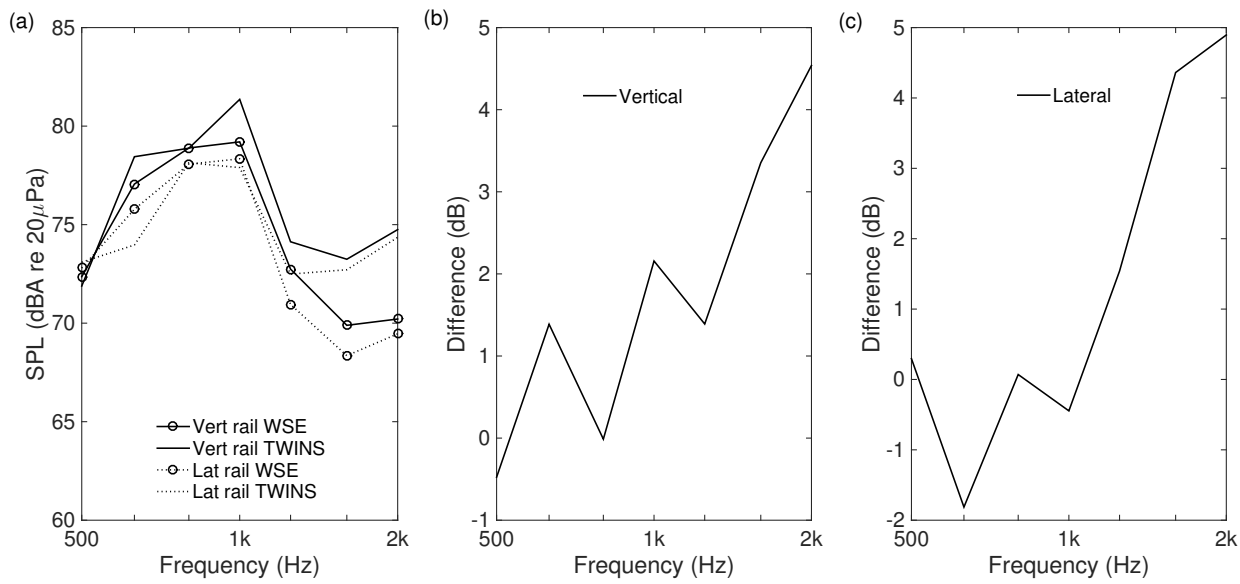

Figure 18: (a) Sound pressure level spectra of the vertical and lateral rail contributions for the train running at $80 \mathrm{~km} / \mathrm{h}$, predicted by TWINS and the WSE method. (b) Difference between WSE and TWINS results of vertical rail contribution. (c) Difference between WSE and TWINS results of lateral rail contribution.
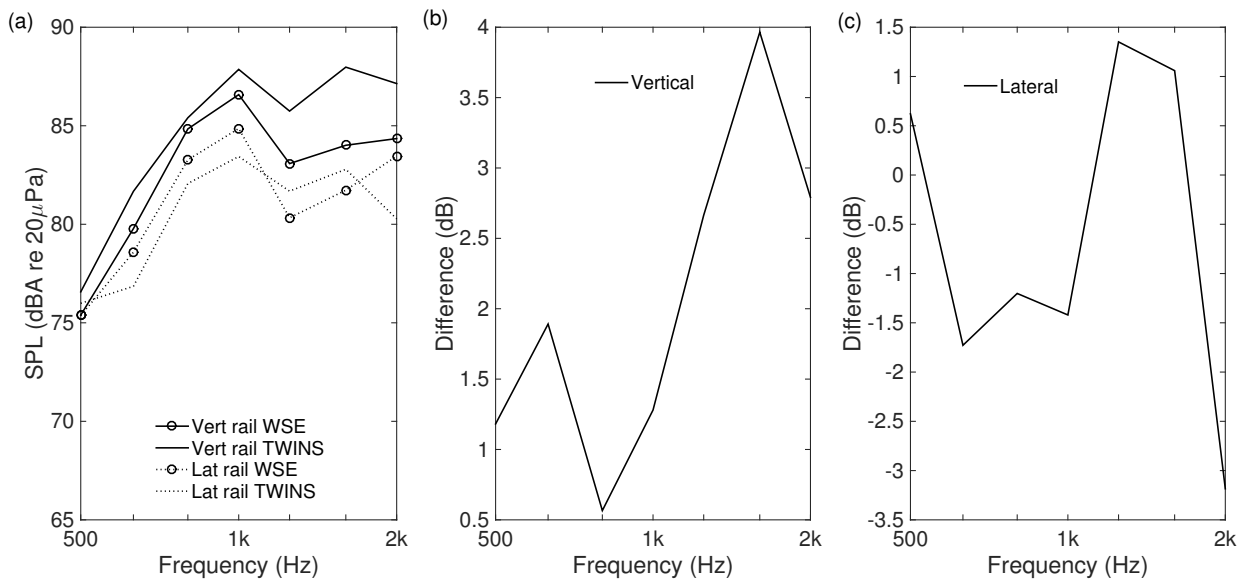

Figure 19: (a) Sound pressure level spectra of the vertical and lateral rail contributions for the train running at $160 \mathrm{~km} / \mathrm{h}$, predicted by TWINS and the WSE method. (b) Difference between WSE and TWINS results of vertical rail contribution. (c) Difference between WSE and TWINS results of lateral rail contribution.

For both velocities we see a good agreement for both the vertical and lateral rail contributions up to $1250 \mathrm{~Hz}$. For higher frequencies one can see a larger discrepancy that, as said earlier, can be attributed to the influence of possible higher order waves that are not included in this analysis, as well as the influence of the wheel contribution. 


\section{Conclusions}

The present paper introduces the wave signature extraction method, whose aim is to separate the rail contribution to pass-by noise in the wavenumber domain. The motivation of this work arises from the problem of railway noise generation, and the compliance of rail-bound vehicles with existing regulations for noise and its environmental impact. The wave signature extraction method requires a uniform line microphone array and two accelerometers, although it can be extended to two-dimensional microphone arrays. The novelty of this work lies in the introduction and application of rail wavenumber filters to microphone array measurements of the train pass-by. The applicability of the method is limited to the frequency range in which the rail behaves like a distributed source. The advantage of the method is that no prior knowledge of the structural properties of the track is in principle needed. The sound pressure levels of the total rail, vertical rail and lateral rail contributions to the pass-by are obtained for two train speeds, and compared with reference data provided from TWINS. The comparison demonstrates that the wave signature extraction predicts well the total rail contribution, except at the frequencies at which the wheel contribution dominates the pass-by noise. Nevertheless there is room for application of the method even at frequencies in which the wheel is dominating, by means of using a denser microphone array. At these frequencies care must then be taken of possible discrepancies of the rail signature with and without the train on the track. Lastly, the results concerning vertical and lateral rail contributions are also close to the predictions by TWINS, indicating that the proposed method also has a potential to separate the sound fields radiated by the two rail vibrational components.

\section{Acknowledgments}

The authors would like to acknowledge the anonymous reviewers for their valuable comments and suggestions. This work is part of the Roll2Rail project, which is financially supported by the European Union's Horizon 2020, grant agreement No. 636032. Acknowledgments are also given to Vetenskapsrådet (Swedish Research Council), grant agreements No. 2012-3723 and No. 2015-04258.

\section{Appendix A. Wave signature extraction algorithm}

Here the matrix $\mathbf{S}(\mathbf{t})$ contains the raw data measured at the sensors and $f_{c}$ is the center frequency of the $1 / 3$ octave band. The algorithm outputs the total rail, vertical rail and lateral rail sound pressure levels.

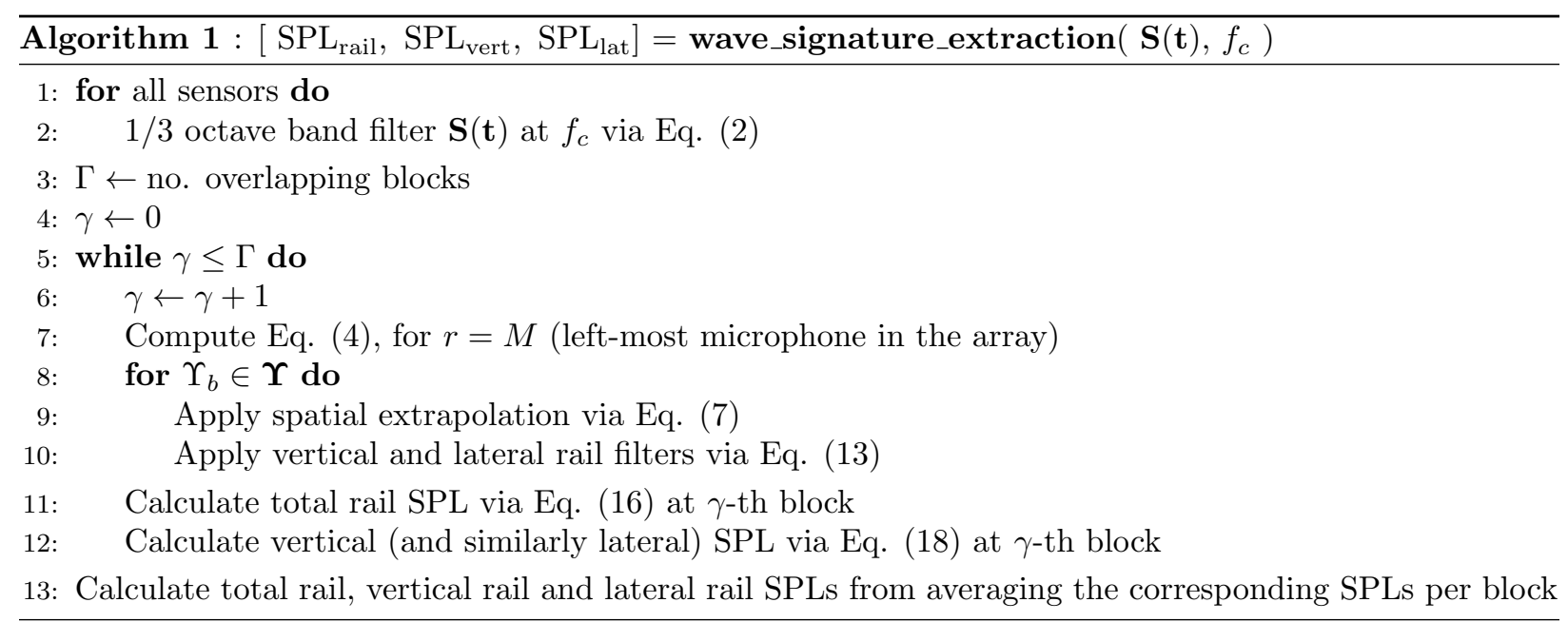




\section{Appendix B. Filter optimization algorithm}

The estimation of the optimal filter at a given frequency band $f_{c}$ is described by the pseudocode below. The recommended choice of filter pass-band $\mathrm{k}_{\mathrm{pb}}$ is between 1 and $3 \mathrm{rad} / \mathrm{m}$.

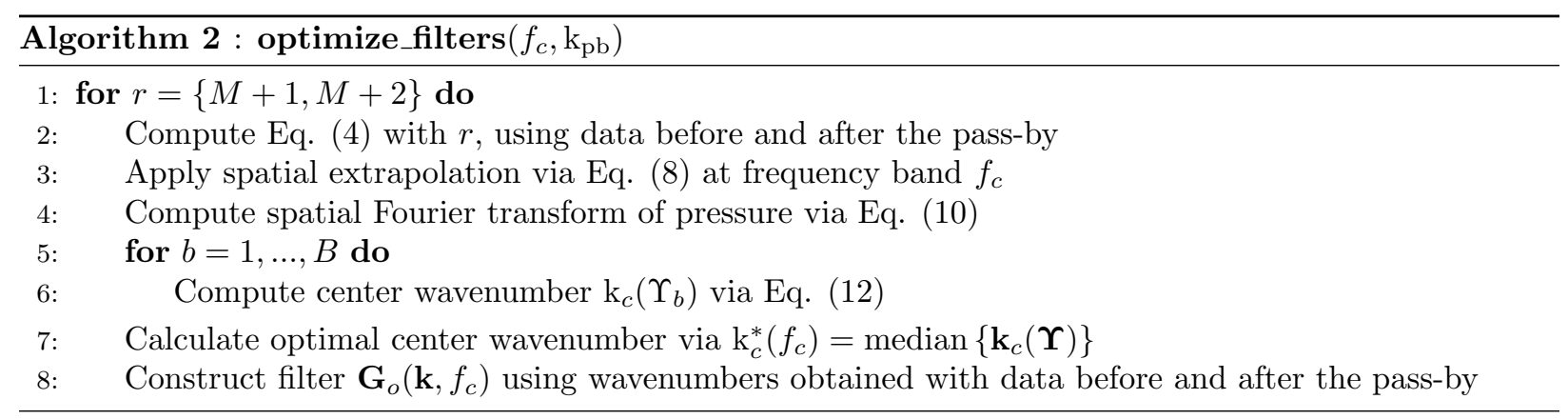

In the second line of the algorithm, the spectra $\mathbf{K}^{(m m)}(\boldsymbol{\Omega})$ and $\mathbf{K}^{(m r)}(\boldsymbol{\Omega})$ are averaged for all data blocks at once via the Welch's method 31 . Also, the time blocks used for the vertical and lateral filters follow the condition on signal power ratio introduced in Eq. (9).

\section{References}

[1] P.-E. Gautier, F. Poisson, F. Letourneaux, High Speed Trains external noise: a review of measurements and source models for the TGV case up to $360 \mathrm{~km} / \mathrm{h}$ in: World Congress Railway Research, Seoul, 2008, pp. 1-10. URL http://uic.org/cdrom/2008/11\{_\}wcrr2008/pdf/S.1.1.4.4.pdf

[2] Commission Regulation (EU) No 1304/2014 of 26 November 2014 on the technical specification for interoperability relating to the subsytem 'rolling stock noise' amending Decision 2008/232/EC and repealing Decision 2011/229/EU Text with EEA relevance (2014).

URL http://data.europa.eu/eli/reg/2014/1304/oj

[3] D. Thompson, P.-E. Gautier, TWINS: a prediction model for wheel-rail rolling noise, in: Inter-noise, Leuven, 1993, pp. $1463-1466$.

[4] D. Thompson, P. Fodiman, H. Mahé, Experimental validation of the TWINS prediction program for rolling noise, Part 2: Results, Journal of Sound and Vibration 193 (1) (1996) 137-147. doi:10.1006/jsvi.1996.0253 URL http://linkinghub.elsevier.com/retrieve/pii/S0022460X96902539

[5] D. Thompson, B. Hemsworth, N. Vincent, Experimental validation of the TWINS prediction program for rolling noise, Par 1: Description of the model and method. Journal of Sound and Vibration 193 (1) (1996) 123-135. doi:10.1006/jsvi. 1996.0252

URL http://linkinghub.elsevier.com/retrieve/pii/S0022460X96902527

[6] E. Bongini, S. Molla, C. Herviou, D. Habault, F. Poisson, Prediction and audio synthesis of vehicle passby noise The Journal of the Acoustical Society of America 123 (5) (2008) 3868-3868. doi:10.1121/1.2935744 URL http://asa.scitation.org/doi/10.1121/1.2935744

[7] E. Bongini, H. Marcus, Railway equivalent noise sources definition: ESM implementation and optimization, in: Acoustics, Nantes, 2012, pp. 173-178. URL https://hal.archives-ouvertes.fr/hal-00810783

[8] F. Letourneaux, O. Coste, C. Mellet, P. Fodiman, Environmental Railway Noise: a Source Separation Measurement Method for Noise Emissions of Vehicles and Track in: Forum Acusticum, Sevilla, 2002, pp. 1-6.

URL http://www. conforg.fr/acoustics2008/cdrom/data/fa2002-sevilla/forumacusticum/archivos/noi03006.pdf

[9] E. Verheijen, M. Paviotti, VTN: A validated method to separate track and vehicle noise and to assess noise reduction measures in: World Congress Railway Research, Edinburgh, 2003, pp. 1-9. URL https://www.dbvision.nl/bestanden/overons/publicaties/2003/VTN\{_\}op\{_\}WCRR2003.pdf

[10] D. Thompson, Sound Radiation from Wheels and Track, in: Railway Noise and Vibration, Elsevier, Oxford, 2009, Ch. 6, pp. 175-222. doi:10.1016/B978-0-08-045147-3.00006-2 URL http://linkinghub.elsevier.com/retrieve/pii/B9780080451473000062

[11] M. Janssens, M. Dittrich, F. de Beer, C. Jones, Railway noise measurement method for pass-by noise, total effective roughness, transfer functions and track spatial decay, Journal of Sound and Vibration 293 (3-5) (2006) 1007-1028. doi: $10.1016 / j \cdot j$ sv.2005.08.070 URL http://linkinghub.elsevier.com/retrieve/pii/S0022460X05007960

[12] ISO 3095 Acoustics - Railway applications - Measurement of noise emitted by railbound vehicles (2013). URL http://www . iso.org/iso/catalogue\{_\}detail.htm?csnumber=55726 
[13] T. Kitagawa, D. Thompson, Comparison of wheel/rail noise radiation on Japanese railways using the TWINS model and microphone array measurements, Journal of Sound and Vibration 293 (3-5) (2006) 496-509. doi:10.1016/j.jsv.2005. 08.037 .

URL http://linkinghub.elsevier.com/retrieve/pii/S0022460X05007558

[14] T. Kitagawa, D. Thompson, The horizontal directivity of noise radiated by a rail and implications for the use of microphone arrays Journal of Sound and Vibration 329 (2) (2010) 202-220. doi:10.1016/j.jsv.2009.09.002

URL http://linkinghub.elsevier.com/retrieve/pii/S0022460X09007020

[15] F. Le Courtois, J.-H. Thomas, F. Poisson, J.-C. Pascal, Identification of the rail radiation using beamforming and a $2 \mathrm{D}$ array, in: Acoustics, Nantes, 2012, pp. 3745-3750.

URL http://hal .archives-ouvertes.fr/hal-00810921/

[16] B. Faure, O. Chiello, M.-A. Pallas, C. Servière, Characterisation of the acoustic field radiated by a rail with a microphone array: The SWEAM method, Journal of Sound and Vibration 346 (2015) 165-190. doi:10.1016/j.jsv.2015.02.022 URL http://linkinghub.elsevier.com/retrieve/pii/S0022460X1500156X

[17] J. D. Maynard, E. G. Williams, Y. Lee, Nearfield acoustic holography: I. Theory of generalized holography and the development of NAH. The Journal of the Acoustical Society of America 78 (4) (1985) 1395-1413. doi:10.1121/1.392911 URL http://asa.scitation.org/doi/10.1121/1.392911

[18] E. G. Williams, Fourier Acoustics: Sound Radiation and Nearfield Acoustical Holography, Academic Press, San Diego, 1999.

[19] P. Kudela, M. Radzieński, W. Ostachowicz, Identification of cracks in thin-walled structures by means of wavenumber filtering Mechanical Systems and Signal Processing 50-51 (2015) 456-466. doi:10.1016/j.ymssp. 2014.05.041 URL http://linkinghub.elsevier.com/retrieve/pii/S0888327014002179

[20] Y. Ren, L. Qiu, S. Yuan, Z. Su, A diagnostic imaging approach for online characterization of multi-impact in aircraft composite structures based on a scanning spatial-wavenumber filter of guided wave, Mechanical Systems and Signal Processing 90 (2017) 44-63. doi:10.1016/j.ymssp.2016.12.005 URL http://linkinghub.elsevier.com/retrieve/pii/S0888327016305180

[21] E. Zea, L. Manzari, I. Lopez Arteaga, G. Squicciarini, D. Thompson, Separation of track contribution to pass-by noise by near-field array techniques, in: 22nd International Congress on Acoustics, Buenos Aires, 2016, pp. 1-10.

[22] D. Thompson, Experimental analysis of wave propagation in railway tracks, Journal of Sound and Vibration 203 (5) (1997) 867-888. doi:10.1006/jsvi.1997.0903

URL http://linkinghub.elsevier.com/retrieve/pii/S0022460X97909032

[23] J. W. Cooley, J. W. Tukey, An Algorithm for the Machine Calculation of Complex Fourier Series Mathematics of Computation 19 (1965) 297-301. doi:10.2307/2003354 URL http://www.jstor.org/stable/2003354?origin=crossref

[24] E. G. Williams, Continuation of acoustic near-fields, The Journal of the Acoustical Society of America 113 (3) (2003) 1273-1281. doi:10.1121/1.1528173. URL http://link.aip.org/link/JASMAN/v113/i3/p1273/s1\{\&\}Agg=doi

[25] I. Lopez Arteaga, R. Scholte, H. Nijmeijer,Improved source reconstruction in Fourier-based Near-field Acoustic Holography applied to small apertures Mechanical Systems and Signal Processing 32 (2012) 359-373. doi:10.1016/j.ymssp. 2012. 06.002 URL http://linkinghub.elsevier.com/retrieve/pii/S0888327012002373

[26] P. M. Morse, K. U. Ingard, Theoretical Acoustics, McGraw-Hill, New York, 1968. doi:10.1119/1.1976432

[27] D. Thompson, Track Vibration in: Railway Noise and Vibration, Elsevier, Oxford, 2009, Ch. 3, pp. 29-95. doi:10.1016/ B978-0-08-045147-3.00003-7

URL http://linkinghub.elsevier.com/retrieve/pii/B9780080451473000037

[28] C. Jones, D. Thompson, Extended validation of a theoretical model for railway rolling noise using novel wheel and track designs, Journal of Sound and Vibration 267 (3) (2003) 509-522. doi:10.1016/S0022-460X (03)00711-9 URL http://linkinghub.elsevier.com/retrieve/pii/S0022460X03007119

[29] D. Thompson, N. Vincent, Track Dynamic Behaviour at High Frequencies. Part 1: Theoretical Models and Laboratory Measurements Vehicle System Dynamics 24 (sup1) (1995) 86-99. doi:10.1080/00423119508969617

URL http://www.tandfonline.com/doi/abs/10.1080/00423119508969617

[30] M. Delany, E. Bazley, Acoustical properties of fibrous absorbent materials, Applied Acoustics 3 (2) (1970) $105-116$. doi:10.1016/0003-682X(70)90031-9

URL http://linkinghub.elsevier.com/retrieve/pii/0003682X70900319

[31] P. Welch, The use of fast Fourier transform for the estimation of power spectra: A method based on time averaging over short, modified periodograms IEEE Transactions on Audio and Electroacoustics 15 (2) (1967) 70-73. doi:10.1109/TAU. 1967.1161901

URL http://ieeexplore.ieee.org/document/1161901/ 